\title{
Effect of roasting time and cryogenic milling on the physicochemical characteristics of dried ginseng powder
}

\author{
Hayeong Jeong, Dong Hyeon Park, Han Geuk Seo, Mi-Jung Choi, and Youngjae Cho* \\ Department of Food Science and Biotechnology of Animal Resources, Konkuk University, 120 Neungdong- \\ ro, Gwangjin-gu, Seoul 05029, Korea \\ *Correspondence: E-mail address: moonjae@konkuk.ac.kr Tel.: +82-2-450-3048. Fax: +82-02-455-3726.
}

\begin{abstract}
This study aimed to decrease the particle size of ginseng by roasting and cryogenic milling to increase its water solubility and physiological activity. The samples were roasted for different times (9-21 $\mathrm{min})$ and generated in different sizes $(10-50$, and $>50 \mu \mathrm{m})$. All roasted samples revealed significantly smaller particle sizes than did non-roasted samples based on Sauter mean diameter (D $[3,2], p<0.05)$. Further, the particle sizes of roasted samples decreased till roasting up to $15 \mathrm{~min}$. In terms of the water solubility index (WSI), antioxidant activity, total polyphenol content (TPC), and total polysaccharides according to particle size, 10-20 $\mu \mathrm{m}$-sized samples showed the highest values when compared with $>50 \mu \mathrm{m}$-sized samples. Based on roasting time, WSI values of all samples roasted for up to $15 \mathrm{~min}$ were higher than those of the control (not roasted) $(p<0.05)$. Antioxidant activity and TPC also increased with increasing roasting time. Total polysaccharide content was the highest upon roasting for $15 \mathrm{~min}$ except for the 10-20 $\mu \mathrm{m}$ sample. Ginsenoside content of roasted samples $>20 \mu \mathrm{m}$ size was higher than that of the control (not roasted) except after $15 \mathrm{~min}$ of roasting. Therefore, roasting and cryogenic milling are effective in producing ginseng root powder.
\end{abstract}

Keywords: cryogenic milling, ginseng, particle size, physiological activity, roasting, water solubility

\section{Introduction}

Ginseng (Panax ginseng C.A. Meyer) has been known as general herbal medicine and functional food in south and southeast Asia for over 2,000 years [1]. Ginseng root is more widely used than the leaf and stem of ginseng because of residual pesticides [2]. Ginseng roots also have many bioactive constituents such as ginsenosides, acidic polysaccharides, and phenolic compounds [3-5]. In particular, major ginsenosides (Rg1, Re, Rf, Rb1, Rc, Rb2, Rb3, and $R d)$ and minor ones ((Rg2(S), $\operatorname{Rg} 2(\mathrm{R}), \operatorname{Rg} 3(\mathrm{~S}), \operatorname{Rg} 3(\mathrm{R})$, and $\mathrm{Rh} 1(\mathrm{~S}))$ ) in ginseng roots have many functions including anti-cancer, antioxidation, anti-stress, blood pressure adjustment, and immune system enhancement effects [6-8]. Currently, ginseng products mostly exist in the form of extracts and powders [9]. Ginseng powder forms are more effective than extract forms, because it contains all the bioactive components with hydrophilic, hydrophobic, and amphiphilic properties [9]. However, ginseng powder has problems of low water solubility, which need to be improved.

Roasting is an appropriate pretreatment solution to improve the low solubility of food products. When roasting raw materials, various components are changed by reactions such as decomposition, synthesis, and condensation [10]. This facilitates the dissolution of components contained in food by high temperature [10]. Further, the roasting treatment breaks down plant cell wall and increases intracellular space, resulting in easy extraction of bioactive components and increased water-soluble contents [11-13]. Thus, roasting increases the ginsenoside, browning substance (melanoid), acidic polysaccharide, and antioxidant contents [14-17]. as Along with roasting, this study used a cryogenic (very-low temperature) milling machine to prepare ginseng powder, which is not common in commercial ginseng grinding. Cryogenic milling is a pulverization method that uses a liquid nitrogen bath at $-196^{\circ} \mathrm{C}$. 
Cryogenic milling reduces particle sizes by weakening the cell wall and increases the water solubility index when compared with effects of high temperature milling [18]. Manohar et al (2001) reported that the particle size of Curcuma domestica subjected to cryogenic milling $(8.56 \mu \mathrm{m})$ was smaller when compared with effects of hammer milling $(88.2 \mu \mathrm{m})$ [19]. Cryogenic milling also prevents discoloration and oxidation by high heat [20].

Therefore, this study aimed to investigate the effect of roasting and cryogenic milling on increasing the water solubility and bioactive constituents in ginseng root. Experiments were also performed to compare the physicochemical properties such as particle size, appearance, color, water solubility index (WSI), DPPH scavenging activity, total polyphenol content (TPC), total/acidic polysaccharide content, and ginsenoside content based on particle size and roasting.

\section{Materials and Methods}

\subsection{Ginseng preparation and pretreatment}

Four-year-old dried ginseng roots (Panax ginseng) were purchased from Bio hongsam (Geumsan, Korea). Ginseng roots were cut to approximately $0.5 \mathrm{~cm}$ thickness and the cut ginseng roots $(125 \mathrm{~g})$ were roasted for $9,12,15,18$, and $21 \mathrm{~min}$ at $180^{\circ} \mathrm{C}$ using a roaster (CBR-101; Gene Café, Ansan, Korea).

\subsection{Preparation of roasted ginseng powder}

Roasted ginseng roots were ground using a cryogenic milling machine (SPEX 6875D Freezer/Mill; Spex sampleprep, Metuchen, NJ, USA). The milling machine was pre-cooled for 5 min to reach $-196^{\circ} \mathrm{C}$ before grinding. The ginseng was placed in a vial and milled at 15 cycles per second (CPS, the number of cycles in which the steel impactor moved forward and backward per second) for $3 \mathrm{~min}$. The milling machine was also cooled for $1 \mathrm{~min}$ between repetitions of the milling cycle.

\subsection{Treatments}

Figure 1 presents the cryogenic milling conditions of dried ginseng root. The cryogenic milling condition was set in three stages including the cycles, rate (CPS), and time. The conditions with 1 cycle were based on $5 \mathrm{~min}$ of precooling, $3 \mathrm{~min}$ of milling time, 1 min of cooling time, and 15 CPS milling rate. The ranges of each stage were set to 2-12 cycles, 5-15 CPSs, and 0.5-5 min. After cryogenic milling of ginseng treatments under different conditions, the treatments were classified according to particle size based on the Sauter mean diameter, D [3, 2] (Table 1). In the range of each size, one treatment was selected as a representative condition. The samples were roasted and then ground by cryogenic milling using the selected condition. Roasting as pretreatment step was performed for 9 , $12,15,18$, and $21 \mathrm{~min}$ at $180^{\circ} \mathrm{C}$ (Table 2).

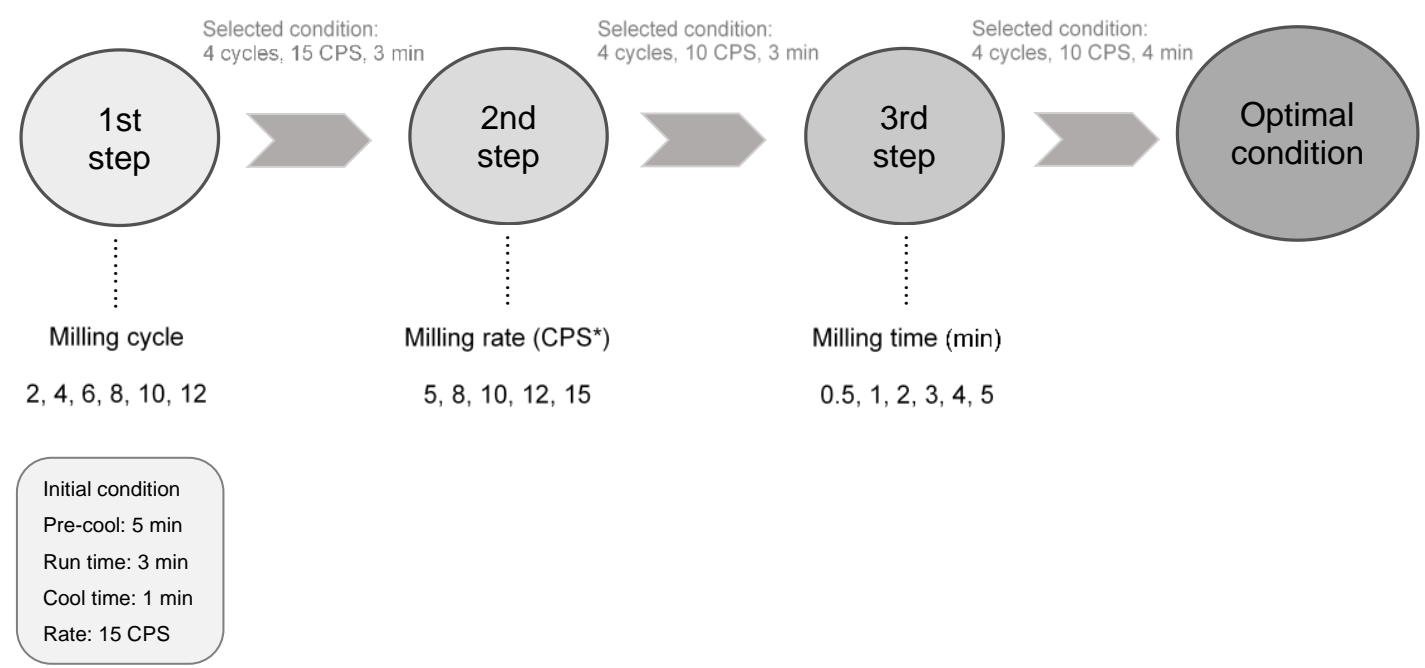

Fig. 1. Cryogenic milling conditions for dried ginseng root. The condition of 1 cycle was 3 minmilling at a rate of 15 CPS. CPS* indicates the number of back-and-forth cycles per second completed by the impactor. 


\subsection{Particle size}

Particle size was measured using a laser diffraction particle size analyzer (Mastersizer 3000; Malvern, Worcestershire, UK). The measurement conditions of particle size were presented in a dry condition as follows: refractive index of particles, 1.520; absorption index of particles, 0.01 ; dispersion medium, dry; dispersion refractive index, 1; particle shape, non-spherical. After placing the ginseng powder on the specimen inlet, the sample was automatically injected using the flow rate ( $50 \%$ power) and the feed rate (50\% power). The particle size of ginseng powders was analyzed at least 5 times.

\subsection{Color}

A petri dish with a size of $35 \times 10 \mathrm{~mm}$ was completely filled with ginseng powders. Color parameters of ginseng powder were measured using a CR-400 colorimeter (Konica minolta sensing, Tokyo, Japan) after calibration with a standard plate ( $L^{*}, 96.67 ; a^{*}, 0.21 ;$ and $\left.b^{*}, 1.82\right)$. All samples were measured 5 times.

\subsection{WSI}

Average WSI of all ginseng powders was analyzed in triplicates as described by Lee et al (2013) [21]. Ginseng powder $(0.5 \mathrm{~g})$ was dissolved in $30 \mathrm{~mL}$ of distilled water, and the solution was shaken for $60 \mathrm{~min}$ at $80^{\circ} \mathrm{C}$ in a water bath (BF-30SB; Biofree, Seoul, Korea). The solution was then centrifuged using a centrifugal separator (1736R; GYROZEN, Daejeon, Korea) for $30 \mathrm{~min}$ at 10,000 rpm. The supernatant was filtered through a Whatman No. 2 paper (Sigma, St. Louis, MO, USA). Next, the filtered solution was dried for $24 \mathrm{~h}$ at $60^{\circ} \mathrm{C}$ using a hot air dryer (LD-918TH; Lequip, Hawsung, Korea). WSI was calculated using the following equation:

WSI $(\%)=($ Weight of dried ginseng solution $/$ Weight of ginseng powder $) \times 100$

\subsection{Antioxidant extract preparation}

Each $1 \mathrm{~g}$ of ginseng sample was extracted with $25 \mathrm{~mL}$ of $70 \%$ ethanol using a water bath (BF30SB; Biofree) for $3 \mathrm{~h}$ at $80^{\circ} \mathrm{C}$. The extracted ginseng solution was filtered through a Whatman No. 2 paper (Sigma) and the filtrate was concentrated using an EYELA rotary evaporator N-1000 (Sunileyela, Seongnam, Korea). The concentrate was then used as a sample after lyophilization using a freeze-dryer (MCFD8512; Ilshinbiobase, Yangju, Korea).

\subsection{DPPH free radical scavenging activity}

Antioxidant activity was measured by modifying the Blois method of removing 2,2-diphenyl-1picryl-hydrazyl (DPPH) (Sigma, St. Louis, MO, USA) free radical [22]. The ginseng solution $(160 \mu \mathrm{L})$ diluted with HPLC-grade methanol was mixed with $40 \mu \mathrm{L}$ of DPPH reagent (Ab 0.95-0.99). In the same way, instead of DPPH reagent, methanol $(40 \mu \mathrm{L})$ was added to the ginseng solution $(160 \mu \mathrm{L})$ for calibration of sample color. The control was prepared by mixing methanol $(160 \mu \mathrm{L})$ and DPPH $(40 \mu \mathrm{L})$. All the samples were reacted at room temperature $\left(22-24^{\circ} \mathrm{C}\right)$ in dark for $10 \mathrm{~min}$. Absorbance of the samples was analyzed at $517 \mathrm{~nm}$ using a Multiskan ${ }^{\mathrm{TM}} \mathrm{GO}$ plate reader (Thermo Scientific, Waltham, MA, USA). The scavenging activity of DPPH free radicals was calculated by the following equation. Next, the antioxidant activity was converted to an IC50 value representing the amount of ginseng that removed $50 \%$ of DPPH free radical.

DPPH free radical scavenging activity $(\%)=[1-\{(\mathrm{A} 1-\mathrm{A} 2) / \mathrm{A} 3\}] \times 100$

A1: Absorbance of DPPH solution $(40 \mu \mathrm{L})+$ ginseng solution $(160 \mu \mathrm{L})$

A2: Absorbance of methanol $(40 \mu \mathrm{L})+$ ginseng solution $(160 \mu \mathrm{L})$

A3: Absorbance of DPPH $(40 \mu \mathrm{L})+$ methanol $(160 \mu \mathrm{L})$

\subsection{Ginseng extract}

To manufacture ginseng extract, $0.5 \mathrm{~g}$ of ginseng powder was dissolved in distilled water (50 $\mathrm{mL}$ ) using a water bath (BF-30SB; Biofree) for $2 \mathrm{~h}$ at $95^{\circ} \mathrm{C}$. Ginseng solutions were then centrifuged using a centrifugal separator (1736R; GYROZEN) for $10 \mathrm{~min}$ at 10,000 rpm and filtered. Then, samples were adjusted to a volumetric flask $(50 \mathrm{~mL})$ for the measurement of physicochemical characteristics (total polyphenol and total acidic polysaccharide contents). 


\subsubsection{Total polyphenol content (TPC)}

TPC was measured using the Folin-Denis method (Ough and Amerine 1980) [23]. Ginseng extract ( $2 \mathrm{~mL}$ ) was vortexed with $2 \mathrm{~mL}$ of Folin-Ciocalteu reagent for $3 \mathrm{~min}$, and $10 \% \mathrm{Na}_{2} \mathrm{CO}_{3}(2 \mathrm{~mL})$ was added to the mixture. The final mixtures were reacted for $1 \mathrm{~h}$ at room temperature in dark, and the sample absorbance was analyzed at $700 \mathrm{~nm}$ using a Multiskan ${ }^{\mathrm{TM}} \mathrm{GO}$ plate reader (Thermo Scientific). The TPC was calculated based on a standard curve prepared using gallic acid (SigmaAldrich, St. Louis, MO, USA).

\subsubsection{Total polysaccharide content}

Total polysaccharide content was analyzed by a method using phenol and sulfuric acid reagents [24]. The extracted ginseng solution $(0.6 \mathrm{~mL})$ was mixed with $5 \%$ phenol $(0.3 \mathrm{~mL})$ using a vortex mixer, and $1.5 \mathrm{~mL}$ concentrated sulfuric acid was added. The mixed solution was shaken for $30 \mathrm{~min}$ at $85^{\circ} \mathrm{C}$ in a water bath (BF-30SB; Biofree), and cooled for $5 \mathrm{~min}$ at room temperature in the dark. Absorbance of the final mixture $(0.2 \mathrm{~mL})$ was analyzed at $490 \mathrm{~nm}$ using a Multiskan ${ }^{\mathrm{TM}} \mathrm{GO}$ plate reader (Thermo Scientific). The total polysaccharide content was obtained using a standard curve prepared with D-glucose (Samchun, Seoul, Korea).

\subsubsection{Acidic polysaccharide content}

The acidic polysaccharide content was analyzed using carbazole and sulfuric acid reagents [25]. The extracted ginseng solution $(0.5 \mathrm{~mL})$ was mixed with $0.25 \mathrm{~mL}$ of carbazole-ethanol reagent (Alfa Aesar, Haverhill, MA, USA), and then with $3 \mathrm{~mL}$ of $\mathrm{H}_{2} \mathrm{SO}_{4}$. The mixed solution was shaken for $5 \mathrm{~min}$ at $80^{\circ} \mathrm{C}$ in a water bath (BF-30SB; Biofree), and cooled for $15 \mathrm{~min}$ in dark at room temperature. Absorbance of the final mixture $(0.2 \mathrm{~mL})$ was then analyzed at $525 \mathrm{~nm}$. The acidic polysaccharide content of ginseng was determined using a standard curve prepared with D-galacturonic acid (Sigma, St. Louis, MO, USA).

\subsection{Ginsenoside content}

After mixing $2 \mathrm{~g}$ of ginseng powder with $70 \%$ methanol $(25 \mathrm{~mL})$, crude ginsenoside was extracted using a Funnel Shaker (RS-1; Jeio Tech, Dajeon, Korea). The extract was adjusted to $50 \mathrm{~mL}$ using a volumetric flask. The ginseng solution was then centrifuged at 3,500 rpm for $5 \mathrm{~min}$ and filtered with a $0.22 \mu \mathrm{m}$ syringe filter (Millipore, Burlington, MA, USA). The filtered ginseng solution was subjected to high-performance liquid chromatography (1260 Infinity 11 LC System; Agilent, Santa Clara, CA, USA) with a C18 HPLC column (Kinetex, $5 \mu \mathrm{m}, 250 \times 4.6 \mathrm{~mm}$; Phenomenex, Seoul, Korea). The temperature condition of the column was set to $30^{\circ} \mathrm{C}$ and the flow rate was $1.0 \mathrm{~mL} / \mathrm{min}$. The ginsenoside content was measured by solvent gradient analysis with water and acetonitrile, and the content of acetonitrile was set as follows: (0-5 min, 20\%; $20 \mathrm{~min}, 23 \%$; $25 \mathrm{~min}, 30 \%$; $45 \mathrm{~min}, 40 \%$; 55 $65 \mathrm{~min}, 50 \%$; and $70-75 \mathrm{~min}, 20 \%$ ). The total operating time was $75 \mathrm{~min}$. The injection volume of the sample was $10 \mu \mathrm{L}$. The sample absorbance was measured at $203 \mathrm{~nm}$.

\subsection{Statistical analysis}

All analyses of ginseng powder were conducted at least 3 times. All result values were indicated as the average value \pm standard deviation using the software SPSS 22.0 (SPSS Inc., USA). Verification of significant differences was performed using Duncan's multiple range test $(p<0.05)$ after one-way analysis of variance (ANOVA).

\section{Results and Discussion}

\subsection{Particle size}

In our preliminary study, the optimal particle size of ginseng was obtained with wet milling and cryogenic milling, respectively. The particle size of ginseng powder ground using wet milling was $114 \mu \mathrm{m}$ based on the $\mathrm{D}[3,2]$ value (data not shown). In contrast, the particle size of ginseng powder using cryogenic milling was significantly decreased to $14 \mu \mathrm{m}$ based on the $\mathrm{D}[3,2]$ value $(p<0.05)$. This result was in agreement with a previous study showing that the particle sizes of ginseng roots ground using wet milling and dry milling were $139.3 \mu \mathrm{m}$ and $15 \mu \mathrm{m}$, respectively, under optimum conditions [26]. Therefore, cryogenic milling was used in this study since particle size reduction was found to be effective. Cryogenic milling a dry milling process, involving grinding at ultra-low temperatures. It increases the hardness and decreases the extensibility of the ginseng cell wall, thus 
making it fragile, which results in rapid particle size reduction [18]. In this study, ginseng powders of different sizes were prepared using different conditions of cycle, rate (CPS), and milling time in a cryogenic milling machine (Table 1$)$. Then, the five representative samples $(1,6,11,14$, and 15) were chosen for each size group from 15 ginseng samples with different sizes, and the chosen samples were roasted for different times. In this study, roasting temperature was $180^{\circ} \mathrm{C}$, which was the optimal temperature in preliminary experiments conducted at various temperatures $(160,170,180$, 190 , and $200^{\circ} \mathrm{C}$ ). Therefore, roasting was performed for 9, 12, 15, 18, and $21 \mathrm{~min}$ according to the roasting time based on $180^{\circ} \mathrm{C}$. Table 2 presents the particle sizes of ginseng powder samples with different roasting time. All roasted treatments demonstrated significant size reduction compared with the control (not roasted) based on the $\mathrm{D}[3,2]$ value $(p<0.05)$. This result was in agreement with the previous study that roasted ginseng powder (below $30.0 \mu \mathrm{m}$ ) had a smaller particle size than that of non-roasted ginseng powder $(1,000-1,250 \mu \mathrm{m})$ [18]. Kim et al. (2004) reported that roasting weakens the starch-protein interactions and destroys the starch granules, eventually reducing the particle size [26]. As the roasting time increased, a decreasing pattern of particle sizes was observed up to $15 \mathrm{~min}$. Im et al (2010) also showed that the initial particle size $(177.2 \mu \mathrm{m})$ was decreased with increasing milling times $(10,20,30$, and $40 \mathrm{~min}$ ), which finally decreased to $92.2 \mu \mathrm{m}$ after $40 \mathrm{~min}$ of grinding [27]. However, when the all roasted treatments were roasted for over $18 \mathrm{~min}$, the particle size was found to be increased again in this study. These results were consistent with previous studies showing that flocculation of powder occurred as the particle size decreased $[28,29]$. The flocculation seemed to arise due to Van der Waals forces produced by increased surface area and energy [30]. However, the particle size of all roasted ginseng samples was smaller than the control based on the $\mathrm{D}[3,2]$ value $(p<0.05)$. Therefore, roasting seemed to be an effective pretreatment for size reduction.

Table 1. Treatment of dried ginseng powder by particle size with various cryogenic milling conditions

\begin{tabular}{|c|c|c|c|c|c|c|c|c|c|}
\hline \multirow[b]{2}{*}{$\begin{array}{l}\text { Size } \\
(\mu \mathrm{m})\end{array}$} & \multirow[b]{2}{*}{ Treatment } & \multicolumn{3}{|c|}{ Treatment conditions } & \multicolumn{5}{|c|}{ Particle size $(\mu \mathrm{m})$} \\
\hline & & Cycle & CPS & Time & $\mathrm{D}[3,2]$ & $\mathrm{D}[4,3]$ & Dx (10) & Dx (50) & Dx (90) \\
\hline \multirow{3}{*}{$10-20$} & 1 & 4 & 10 & 4 & $14.0 \pm 0.2^{\mathrm{j} C}$ & $68.20 \pm 7.10^{\mathrm{iB}}$ & $5.80 \pm 0.2^{\mathrm{jB}}$ & $28.50 \pm 0.9 \mathrm{gC}$ & $145.9 \pm 8.50^{\mathrm{iB}}$ \\
\hline & 2 & 4 & 10 & 3 & $15.0 \pm 0.7 \mathrm{jB}$ & $63.80 \pm 4.50^{\mathrm{iB}}$ & $6.10 \pm 0.3 \mathrm{j}^{\mathrm{A}}$ & $30.80 \pm 1.2^{\mathrm{gB}}$ & $150.9 \pm 8.10^{\mathrm{iв}}$ \\
\hline & 3 & 4 & 10 & 5 & $15.5 \pm 0.6^{\mathrm{jA}}$ & $89.40 \pm 10.9^{\mathrm{hA}}$ & $6.20 \pm 0.2^{\mathrm{jA}}$ & $32.10 \pm 1.7 \mathrm{gA}$ & $193.5 \pm 16.5^{\mathrm{hA}}$ \\
\hline \multirow{5}{*}{$20-30$} & 4 & 4 & 10 & 2 & $21.0 \pm 2.2^{\mathrm{iE}}$ & $114.4 \pm 5.50^{\mathrm{fgBC}}$ & $8.10 \pm 0.9$ iE & $47.60 \pm 1.4^{\mathrm{fC}}$ & $308.4 \pm 15.7^{\mathrm{fC}}$ \\
\hline & 5 & 4 & 8 & 3 & $22.5 \pm 0.4^{\mathrm{hD}}$ & $105.1 \pm 3.70 \mathrm{gC}$ & $8.90 \pm 0.3^{\mathrm{hD}}$ & $47.80 \pm 0.8^{\mathrm{fC}}$ & $263.8 \pm 11.1 \mathrm{gD}$ \\
\hline & 6 & 4 & 12 & 3 & $24.9 \pm 0.4 \mathrm{gC}$ & $113.7 \pm 4.20^{\mathrm{fgBC}}$ & $9.60 \pm 0.1 \mathrm{gC}$ & $57.20 \pm 0.8^{\mathrm{eB}}$ & $299.2 \pm 4.70^{\mathrm{fCD}}$ \\
\hline & 7 & 6 & 15 & 3 & $28.7 \pm 1.0^{\mathrm{eA}}$ & $131.5 \pm 18.0^{\mathrm{eA}}$ & $11.8 \pm 0.3^{\mathrm{eB}}$ & $53.70 \pm 3.5^{\mathrm{eA}}$ & $377.9 \pm 78.2^{\mathrm{dA}}$ \\
\hline & 8 & 4 & 15 & 3 & $30.1 \pm 1.1^{\mathrm{fB}}$ & $124.0 \pm 8.20^{\mathrm{fB}}$ & $13.8 \pm 0.2^{\mathrm{dA}}$ & $60.50 \pm 2.6^{\mathrm{eA}}$ & $345.5 \pm 40.4^{\mathrm{eB}}$ \\
\hline \multirow{5}{*}{$30-40$} & 9 & 8 & 15 & 3 & $31.7 \pm 1.2^{\mathrm{eC}}$ & $162.8 \pm 17.2^{\mathrm{eC}}$ & $12.9 \pm 0.3^{\mathrm{eB}}$ & $60.80 \pm 4.7^{\mathrm{eC}}$ & $522.3 \pm 54.0^{\mathrm{dC}}$ \\
\hline & 10 & 10 & 15 & 3 & $31.9 \pm 1.6^{\mathrm{eC}}$ & $177.0 \pm 14.4^{\mathrm{dB}}$ & $11.9 \pm 0.3^{\mathrm{fD}}$ & $61.10 \pm 4.3^{\mathrm{eC}}$ & $567.3 \pm 45.4^{\text {св }}$ \\
\hline & 11 & 4 & 10 & 1 & $34.5 \pm 0.8^{\mathrm{dB}}$ & $215.5 \pm 11.2^{\mathrm{bA}}$ & $12.4 \pm 0.3^{\mathrm{eC}}$ & $113.8 \pm 9.1^{\mathrm{bA}}$ & $591.7 \pm 24.5^{\mathrm{bcB}}$ \\
\hline & 12 & 12 & 15 & 3 & $40.0 \pm 2.8^{\mathrm{cA}}$ & $226.0 \pm 3.00^{\mathrm{bA}}$ & $15.2 \pm 0.5^{\mathrm{cA}}$ & $86.70 \pm 6.3^{\mathrm{dB}}$ & $664.2 \pm 33.0^{\mathrm{aA}}$ \\
\hline & 13 & 4 & 5 & 3 & $40.6 \pm 1.0^{\mathrm{cA}}$ & $217.6 \pm 6.40^{\mathrm{bA}}$ & $15.0 \pm 0.3^{\mathrm{cA}}$ & $111.3 \pm 4.4^{\mathrm{bA}}$ & $602.4 \pm 15.8^{\mathrm{bB}}$ \\
\hline $40-50$ & 14 & 2 & 15 & 3 & $46.8 \pm 1.0^{\mathrm{b}}$ & $198.8 \pm 7.00^{c}$ & $19.0 \pm 0.4^{\mathrm{b}}$ & $104.0 \pm 1.4^{\mathrm{c}}$ & $529.4 \pm 22.8^{\mathrm{d}}$ \\
\hline$>50$ & 15 & 4 & 10 & 0.5 & $64.1 \pm 5.3^{\mathrm{a}}$ & $289.0 \pm 5.00^{\mathrm{a}}$ & $25.0 \pm 2.1^{\mathrm{a}}$ & $207.4 \pm 6.3^{a}$ & $690.4 \pm 10.6^{a}$ \\
\hline
\end{tabular}

a-jMeans with different superscripts within whole particle size group are significantly different $(p<$ $0.05)$. A-EMeans with different superscripts within the similar size group are significantly different $(p$ $<0.05)$. Particle size $(\mu \mathrm{m})$ set up based on $\mathrm{D}[3,2]$. Fifteen treatments were generated according to cycle, CPS (the number of back-and-forth cycles per second), and time conditions, and the larger the number of treatments, the larger is the particle size. One cycle is based on pulverization for $3 \mathrm{~min}$ at a rate of 15 CPS oscillating 15 times per second. 
Table 2. Particle size of dried ginseng powder with various roasting time conditions

\begin{tabular}{|c|c|c|c|c|c|c|}
\hline \multirow[b]{2}{*}{$\begin{array}{l}\text { Size } \\
(\mu \mathrm{m})\end{array}$} & \multirow{2}{*}{$\begin{array}{l}\text { Roasting }{ }^{1)} \\
\text { Time } \\
(\mathrm{min}) \\
\end{array}$} & \multicolumn{5}{|c|}{ Particle size $(\mu \mathrm{m})$} \\
\hline & & $\mathrm{D}[3,2]$ & $\mathrm{D}[4,3]$ & Dx (10) & Dx (50) & Dx (90) \\
\hline \multirow{6}{*}{$10-20$} & Control & $14.00 \pm 0.20^{\mathrm{eA}}$ & $68.20 \pm 7.10^{\mathrm{eA}}$ & $5.80 \pm 0.20^{\mathrm{eA}}$ & $28.50 \pm 0.90^{\mathrm{eA}}$ & $145.90 \pm 8.50^{\mathrm{eA}}$ \\
\hline & 9 & $8.22 \pm 0.33^{\mathrm{eC}}$ & $45.78 \pm 4.61^{\mathrm{eB}}$ & $3.28 \pm 0.11^{\mathrm{eC}}$ & $18.67 \pm 1.70^{\mathrm{eв}}$ & $110.00 \pm 3.92^{\mathrm{dB}}$ \\
\hline & 12 & $7.58 \pm 0.27^{\mathrm{dD}}$ & $30.83 \pm 2.86^{\mathrm{eC}}$ & $3.04 \pm 0.10^{\mathrm{eD}}$ & $15.87 \pm 1.13^{\mathrm{eC}}$ & $77.60 \pm 8.60^{\mathrm{dC}}$ \\
\hline & 15 & $5.60 \pm 0.12^{\mathrm{eF}}$ & $20.94 \pm 0.88^{\mathrm{dD}}$ & $2.19 \pm 0.05^{\mathrm{eE}}$ & $10.29 \pm 0.63^{\mathrm{eE}}$ & $55.83 \pm 1.18^{\mathrm{eD}}$ \\
\hline & 18 & $7.06 \pm 0.10^{\mathrm{eE}}$ & $26.03 \pm 2.02^{\mathrm{eCD}}$ & $2.90 \pm 0.07 \mathrm{eD}$ & $13.93 \pm 0.30^{\mathrm{eD}}$ & $68.48 \pm 6.00^{\mathrm{dC}}$ \\
\hline & 21 & $9.27 \pm 0.10^{\mathrm{dB}}$ & $29.38 \pm 3.99 \mathrm{dC}$ & $3.89 \pm 0.05^{\mathrm{dB}}$ & $17.77 \pm 1.67^{\mathrm{bB}}$ & $70.55 \pm 11.40^{\mathrm{dC}}$ \\
\hline \multirow{6}{*}{$20-30$} & Control & $24.90 \pm 0.40^{\mathrm{dA}}$ & $113.70 \pm 4.20^{\mathrm{dA}}$ & $9.60 \pm 0.10^{\mathrm{dA}}$ & $57.20 \pm 0.80^{\mathrm{dA}}$ & $299.20 \pm 4.70^{\mathrm{dA}}$ \\
\hline & 9 & $20.34 \pm 1.52^{\mathrm{dB}}$ & $112.50 \pm 5.45^{\mathrm{dA}}$ & $7.87 \pm 0.15^{\mathrm{dB}}$ & $48.94 \pm 1.14^{\mathrm{dB}}$ & $288.33 \pm 5.03^{\mathrm{cAB}}$ \\
\hline & 12 & $16.92 \pm 0.63^{\mathrm{cD}}$ & $92.02 \pm 13.90^{\mathrm{dB}}$ & $7.10 \pm 0.34^{\mathrm{cC}}$ & $35.08 \pm 0.30^{\mathrm{dC}}$ & $274.50 \pm 19.94^{\text {св }}$ \\
\hline & 15 & $9.80 \pm 0.44^{\mathrm{dE}}$ & $52.40 \pm 11.92^{\mathrm{cD}}$ & $3.87 \pm 0.13^{\mathrm{dE}}$ & $22.56 \pm 2.38^{\mathrm{dD}}$ & $118.35 \pm 16.78^{\mathrm{dE}}$ \\
\hline & 18 & $17.38 \pm 0.39 \mathrm{dCD}$ & $68.33 \pm 5.95^{\mathrm{dC}}$ & $6.51 \pm 0.69 \mathrm{dD}$ & $35.58 \pm 6.31^{\mathrm{dC}}$ & $181.33 \pm 14.64^{\mathrm{cD}}$ \\
\hline & 21 & $17.62 \pm 0.48^{\mathrm{cC}}$ & $97.50 \pm 8.48^{\mathrm{cB}}$ & $7.07 \pm 0.24^{\mathrm{cC}}$ & $37.62 \pm 1.48^{\mathrm{bC}}$ & $252.67 \pm 4.16^{\mathrm{cC}}$ \\
\hline \multirow{6}{*}{$30-40$} & Control & $34.50 \pm 0.80^{\mathrm{cA}}$ & $215.50 \pm 11.20^{\mathrm{bA}}$ & $12.40 \pm 0.30^{\mathrm{cA}}$ & $113.80 \pm 9.10^{\mathrm{bA}}$ & $591.70 \pm 24.50^{\mathrm{bA}}$ \\
\hline & 9 & $30.60 \pm 0.84^{\mathrm{cB}}$ & $181.00 \pm 10.86^{\mathrm{cB}}$ & $11.88 \pm 0.44^{\mathrm{cB}}$ & $87.08 \pm 1.99 \mathrm{cB}$ & $503.00 \pm 46.90^{\text {bв }}$ \\
\hline & 12 & $16.45 \pm 0.13^{\mathrm{cD}}$ & $137.40 \pm 9.10^{\mathrm{cD}}$ & $6.38 \pm 0.12^{\mathrm{dD}}$ & $48.70 \pm 1.21^{\mathrm{cD}}$ & $403.17 \pm 57.68^{\mathrm{bC}}$ \\
\hline & 15 & $12.66 \pm 0.59^{\mathrm{cE}}$ & $62.40 \pm 2.49^{\mathrm{cE}}$ & $4.92 \pm 0.16^{\mathrm{cE}}$ & $31.21 \pm 2.28^{\mathrm{cE}}$ & $180.80 \pm 6.30^{\mathrm{cD}}$ \\
\hline & 18 & $23.58 \pm 0.99 \propto c$ & $143.75 \pm 3.86^{\mathrm{cCD}}$ & $9.26 \pm 0.48^{\mathrm{cc}}$ & $57.28 \pm 2.61^{\mathrm{cc}}$ & $403.20 \pm 28.90^{\mathrm{bC}}$ \\
\hline & 21 & $24.22 \pm 0.79 \mathrm{bC}$ & $155.00 \pm 3.56^{\mathrm{bC}}$ & $9.27 \pm 0.46^{\mathrm{bcc}}$ & $62.08 \pm 1.65^{\mathrm{bC}}$ & $445.20 \pm 34.80^{\mathrm{bC}}$ \\
\hline \multirow{6}{*}{$40-50$} & Control & $46.80 \pm 1.00^{\mathrm{bA}}$ & $198.80 \pm 7.00^{\mathrm{cA}}$ & $19.00 \pm 0.40^{\mathrm{bA}}$ & $104.00 \pm 1.40^{\mathrm{cB}}$ & $529.40 \pm 22.80^{\mathrm{cA}}$ \\
\hline & 9 & $37.98 \pm 2.72^{\text {bв }}$ & $208.67 \pm 6.81^{\mathrm{bA}}$ & $14.18 \pm 1.18^{\mathrm{bB}}$ & $127.25 \pm 11.76^{\mathrm{bA}}$ & $524.67 \pm 15.50^{\mathrm{bA}}$ \\
\hline & 12 & $28.80 \pm 0.73^{\mathrm{bC}}$ & $156.60 \pm 3.78^{\mathrm{bB}}$ & $11.14 \pm 0.46^{\mathrm{bC}}$ & $75.54 \pm 1.84^{\mathrm{bC}}$ & $423.80 \pm 15.91^{\mathrm{bB}}$ \\
\hline & 15 & $14.88 \pm 0.53^{\mathrm{bE}}$ & $90.56 \pm 11.83^{\mathrm{bc}}$ & $5.98 \pm 0.28^{\mathrm{bE}}$ & $41.40 \pm 0.88^{\mathrm{bD}}$ & $248.50 \pm 29.22^{\mathrm{bc}}$ \\
\hline & 18 & $26.83 \pm 0.68^{\mathrm{bD}}$ & $156.00 \pm 3.87^{\mathrm{bB}}$ & $10.36 \pm 0.46^{\mathrm{bD}}$ & $73.58 \pm 3.24^{\mathrm{bC}}$ & $427.00 \pm 8.03^{\mathrm{bB}}$ \\
\hline & 21 & $26.46 \pm 0.50^{\mathrm{bD}}$ & $157.40 \pm 4.28^{\mathrm{bB}}$ & $10.05 \pm 0.32^{\mathrm{bD}}$ & $70.20 \pm 1.90^{\mathrm{bC}}$ & $442.00 \pm 18.75^{\mathrm{bB}}$ \\
\hline \multirow{6}{*}{$>50$} & Control & $64.10 \pm 5.30^{\mathrm{aA}}$ & $289.00 \pm 5.00^{\mathrm{aB}}$ & $25.00 \pm 2.10^{\mathrm{aA}}$ & $207.40 \pm 6.30^{\mathrm{aAB}}$ & $690.40 \pm 10.60^{\mathrm{aB}}$ \\
\hline & 9 & $48.33 \pm 0.96^{\mathrm{aB}}$ & $282.25 \pm 10.14^{\mathrm{aB}}$ & $18.80 \pm 0.75^{\mathrm{aB}}$ & $193.50 \pm 7.33^{\mathrm{aв}}$ & $686.00 \pm 26.15^{\mathrm{aB}}$ \\
\hline & 12 & $45.83 \pm 0.51^{\mathrm{aB}}$ & $287.67 \pm 0.58^{\mathrm{aB}}$ & $16.53 \pm 0.38^{\mathrm{aB}}$ & $197.75 \pm 7.14^{\mathrm{aAB}}$ & $708.67 \pm 7.77^{\mathrm{aB}}$ \\
\hline & 15 & $25.58 \pm 0.10^{\mathrm{ac}}$ & $219.40 \pm 4.28^{\mathrm{aC}}$ & $9.73 \pm 0.12^{\mathrm{aC}}$ & $96.60 \pm 3.32^{\mathrm{aC}}$ & $635.20 \pm 10.28^{\mathrm{aC}}$ \\
\hline & 18 & $43.26 \pm 1.31^{\mathrm{aB}}$ & $293.80 \pm 14.82^{\mathrm{aB}}$ & $15.76 \pm 0.64^{\mathrm{aв}}$ & $192.00 \pm 19.7^{\mathrm{aB}}$ & $731.60 \pm 19.40^{\mathrm{aв}}$ \\
\hline & 21 & $45.96 \pm 6.74^{\mathrm{aB}}$ & $338.57 \pm 57.82^{\mathrm{aA}}$ & $16.97 \pm 3.84^{\mathrm{aB}}$ & $265.71 \pm 95.90^{\mathrm{aA}}$ & $781.57 \pm 54.01^{\mathrm{aA}}$ \\
\hline
\end{tabular}

a-eMeans with different superscripts within the same time group are significantly different $(p<0.05)$. A-FMeans with different superscripts within the same size group are significantly different $(p<0.05)$. ${ }^{1)}$ After cryogenic milling of ginseng samples with different conditions, the samples were classified with by their particle sizes. One treatment condition in each size range was then selected, and samples selected by particle size were roasted and pulverized by cryogenic milling.

\subsection{Appearance}

The appearances of dried raw ginseng are shown in Figure 2 to compare the changes before and after roasting. As the roasting time increased, the color of ginseng seemed to be gradually changed to black, and started to be burned in case of roasting over $18 \mathrm{~min}$. After dried ginseng was pulverized by cryogenic milling, Figure 3 presents the appearance of ginseng powder before and after roasting. According to the particle sizes, the appearance of the control group (not roasted) was rougher as the particle size was large. However, the roasted powders seemed to be softer than the control. According to the roasting times, the color of ginseng powders appeared to be gradually darkened with 
increasing roasting time. The color of ginseng powder darkened as the particle size increased and the roasting time increased.

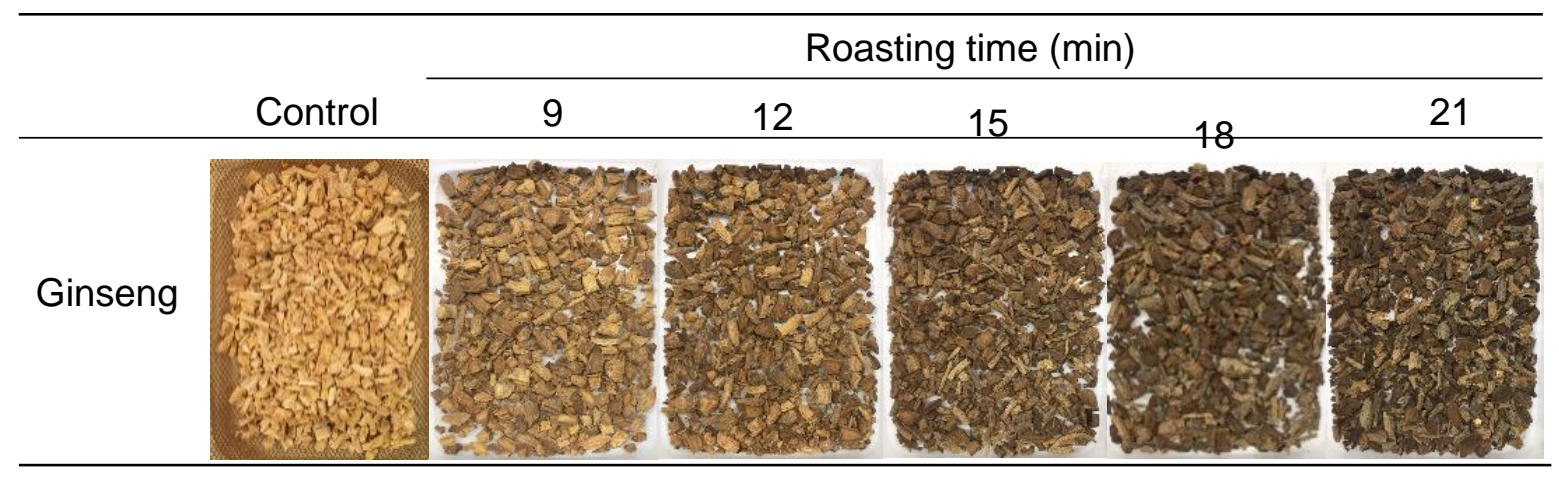

Fig. 2. Appearance of dried ginseng root roasted for different times.

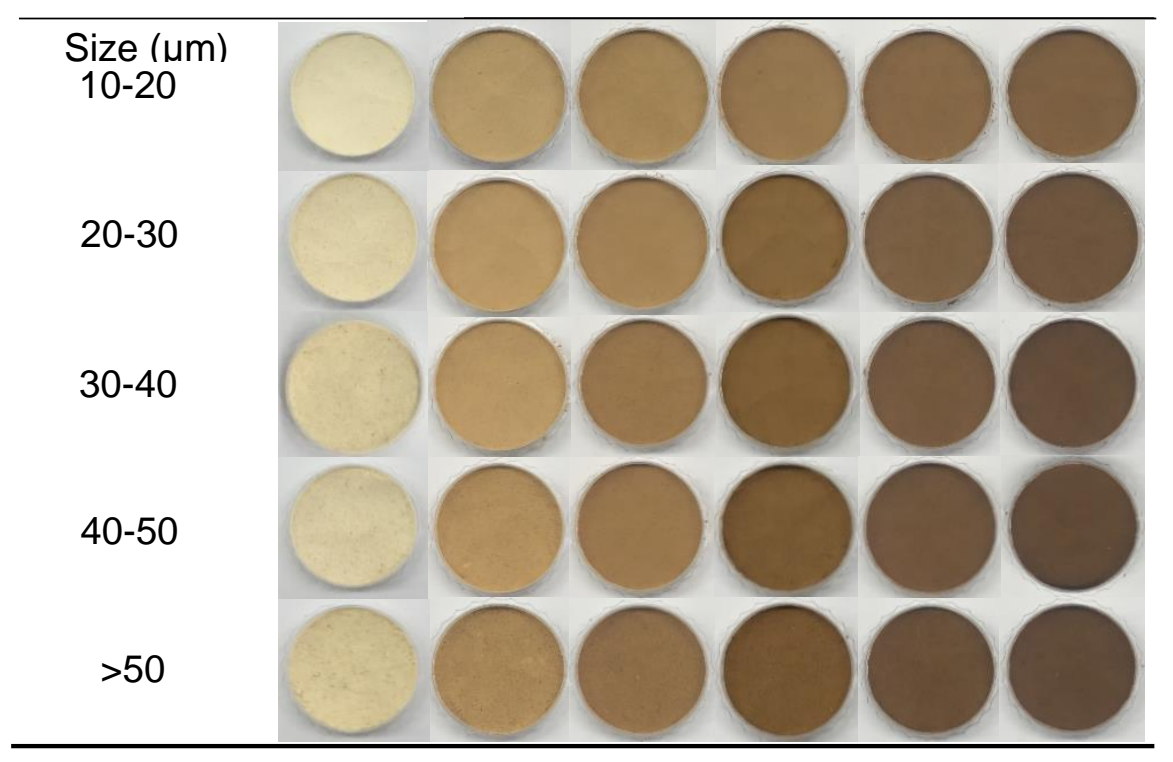

Fig. 3. Appearance of dried ginseng root powder pretreated with different roasting times.

\subsection{Color}

Color changes in ginseng powder were observed according to particle size and roasting time (Table 3). When comparing with the control alone as the particle size increased, the color values showed lower lightness $\left(\mathrm{L}^{*}\right)$ and higher redness $\left(\mathrm{a}^{*}\right)$ and yellowness $\left(\mathrm{b}^{*}\right)$. All color values of controls were significantly different except for the $\mathrm{a}^{*}$ and $\mathrm{b}^{*}$ values of the samples with sizes $>50 \mu \mathrm{m}(p<0.05)$. Kim et al. (2012) reported that in general, for powders, $L^{*}$ values increased as the particle size decreased, whereas the $a^{*}$ and $b^{*}$ values varied according to the sample [31]. The smaller the particle size, the higher is the scattered light, and thus the higher is the $\mathrm{L}^{*}$ [32]. With the pretreatment of roasting, $\mathrm{L}^{*}$ values of roasted ginseng powders were significantly lower than those of the controls $(p$ $<0.05)$, whereas the $\mathrm{a}^{*}$ values were significantly higher than those of the controls $(p<0.05)$. Further, $\mathrm{b}^{*}$ values were lower than those of the controls when roasting was done for over 12 min except for $10-20 \mu \mathrm{m}$ sized samples $(p<0.05)$. As the roasting time increased in roasted treatments, the $\mathrm{L}^{*}$ and $\mathrm{b}^{*}$ values of all samples were significantly decreased $(p<0.05)$. The $\mathrm{a}^{*}$ values tended to increase until roasting for $12 \mathrm{~min}$. However, the a* values were significantly decreased when samples were roasted for over $15 \mathrm{~min}$ with an exception of $10-20 \mu \mathrm{m}$ sized samples $(p<0.05)$. This result was in agreement 
with a previous study showing that $\mathrm{L}^{*}$ and $\mathrm{b}^{*}$ values tended to decrease as the roasting time (5.27, $6.19,6.39$, and $8.01 \mathrm{~min}$ ) increased at $170^{\circ} \mathrm{C}$ [33]. Further, the $\mathrm{a}^{*}$ values tended to increase at the early stage of roasting and decreased again as the roasting time increased [33]. These color changes were caused by the Maillard reaction of non-enzymatic browning and caramelization [33]. Particle size and roasting time was found to affect the color parameters of ginseng powder.

Table 3. Color of dried ginseng root powder with various roasting temperature conditions

\begin{tabular}{|c|c|c|c|c|c|c|c|c|c|c|}
\hline \multirow[b]{2}{*}{ Size $(\mu \mathrm{m})$} & \multirow{3}{*}{$\begin{array}{c}\text { Roasting }^{1)} \\
\text { Time (min) } \\
\text { Control }\end{array}$} & \multicolumn{8}{|c|}{ Color } & \\
\hline & & \multicolumn{3}{|c|}{$\mathrm{L}^{*}$} & \multicolumn{3}{|c|}{$a^{*}$} & \multicolumn{3}{|c|}{$b^{*}$} \\
\hline \multirow{6}{*}{$10-20$} & & 82.13 & \pm & $0.54^{\mathrm{aA}}$ & 0.71 & \pm & $0.22^{\mathrm{dC}}$ & 15.40 & \pm & $0.14^{\mathrm{dC}}$ \\
\hline & 9 & 65.65 & \pm & $0.04^{\mathrm{aB}}$ & 5.26 & \pm & $0.00^{\mathrm{eB}}$ & 17.03 & \pm & $0.04^{\mathrm{cA}}$ \\
\hline & 12 & 57.65 & \pm & $0.05^{\mathrm{aC}}$ & 6.01 & \pm & $0.02^{\mathrm{eA}}$ & 16.16 & \pm & $0.03^{\mathrm{aB}}$ \\
\hline & 15 & 52.35 & \pm & $0.08^{\mathrm{aD}}$ & 5.44 & \pm & $0.03^{\mathrm{dB}}$ & 14.32 & \pm & $0.06^{\mathrm{aD}}$ \\
\hline & 18 & 45.95 & \pm & $0.07^{\mathrm{aE}}$ & 5.87 & \pm & $0.01^{\mathrm{aA}}$ & 12.36 & \pm & $0.01^{\mathrm{aE}}$ \\
\hline & 21 & 44.53 & \pm & $0.05^{\mathrm{aF}}$ & 5.42 & \pm & $0.01^{\mathrm{aB}}$ & 11.50 & \pm & $0.03^{\mathrm{aF}}$ \\
\hline \multirow{6}{*}{$20-30$} & Control & 80.44 & \pm & $0.25^{\mathrm{bA}}$ & 1.07 & \pm & $0.04^{\mathrm{cF}}$ & 16.03 & \pm & $0.36^{\mathrm{cB}}$ \\
\hline & 9 & 60.99 & \pm & $0.06^{\mathrm{cB}}$ & 5.94 & \pm & $0.01^{\mathrm{dC}}$ & 17.02 & \pm & $0.03^{\mathrm{cA}}$ \\
\hline & 12 & 54.27 & \pm & $0.13^{\mathrm{bC}}$ & 6.29 & \pm & $0.01^{\mathrm{dA}}$ & 15.53 & \pm & $0.03^{\mathrm{bC}}$ \\
\hline & 15 & 44.59 & \pm & $0.09^{\mathrm{bD}}$ & 6.04 & \pm & $0.05^{\mathrm{bB}}$ & 11.90 & \pm & $0.03^{\mathrm{cD}}$ \\
\hline & 18 & 44.03 & \pm & $0.02^{\mathrm{bE}}$ & 5.55 & \pm & $0.02^{\mathrm{cD}}$ & 11.26 & \pm & $0.01^{\mathrm{bE}}$ \\
\hline & 21 & 40.31 & \pm & $0.01^{\mathrm{bF}}$ & 5.27 & \pm & $0.00^{\mathrm{bE}}$ & 9.57 & \pm & $0.00^{\mathrm{bF}}$ \\
\hline \multirow{6}{*}{$30-40$} & Control & 78.77 & \pm & $0.77^{\mathrm{cA}}$ & 1.41 & \pm & $0.09^{\mathrm{bF}}$ & 16.64 & \pm & $0.26^{\mathrm{bB}}$ \\
\hline & 9 & 61.23 & \pm & $0.05^{\mathrm{bB}}$ & 6.01 & \pm & $0.01^{\mathrm{cC}}$ & 17.24 & \pm & $0.01^{\mathrm{aA}}$ \\
\hline & 12 & 51.71 & \pm & $0.14^{\mathrm{cC}}$ & 6.66 & \pm & $0.02^{\mathrm{bA}}$ & 15.08 & \pm & $0.03^{\mathrm{cC}}$ \\
\hline & 15 & 43.83 & \pm & $0.16^{\mathrm{cD}}$ & 6.32 & \pm & $0.02^{\mathrm{aB}}$ & 12.28 & \pm & $0.02^{\mathrm{bD}}$ \\
\hline & 18 & 42.03 & \pm & $0.02^{\mathrm{cE}}$ & 5.71 & \pm & $0.02^{\mathrm{bD}}$ & 10.60 & \pm & $0.02^{\mathrm{cE}}$ \\
\hline & 21 & 38.19 & \pm & $0.13^{\mathrm{cF}}$ & 4.99 & \pm & $0.01^{\mathrm{cE}}$ & 8.22 & \pm & $0.05^{\mathrm{cF}}$ \\
\hline \multirow{6}{*}{$40-50$} & Control & 77.57 & \pm & $0.04^{\mathrm{dA}}$ & 1.83 & \pm & $0.02^{\mathrm{aF}}$ & 17.21 & \pm & $0.01^{\mathrm{aA}}$ \\
\hline & 9 & 59.63 & \pm & $0.08^{\mathrm{dB}}$ & 6.49 & \pm & $0.01^{\mathrm{bB}}$ & 17.18 & \pm & $0.05^{\mathrm{bA}}$ \\
\hline & 12 & 51.65 & \pm & $0.14^{\mathrm{cC}}$ & 6.56 & \pm & $0.03^{\mathrm{cA}}$ & 14.77 & \pm & $0.00^{\mathrm{dB}}$ \\
\hline & 15 & 43.36 & \pm & $0.03^{\mathrm{dD}}$ & 5.91 & \pm & $0.03^{\mathrm{cC}}$ & 11.23 & \pm & $0.05^{\mathrm{dC}}$ \\
\hline & 18 & 40.78 & \pm & $0.14^{\mathrm{dE}}$ & 5.54 & \pm & $0.02^{\mathrm{cD}}$ & 9.81 & \pm & $0.04^{\mathrm{dD}}$ \\
\hline & 21 & 37.72 & \pm & $0.07^{\mathrm{dF}}$ & 4.99 & \pm & $0.02^{\mathrm{cE}}$ & 8.01 & \pm & $0.01^{\mathrm{dE}}$ \\
\hline \multirow{6}{*}{$>50$} & Control & 76.31 & \pm & $0.10^{\mathrm{eA}}$ & 1.91 & \pm & $0.05^{\mathrm{aE}}$ & 17.26 & \pm & $0.01^{\mathrm{aA}}$ \\
\hline & 9 & 56.92 & \pm & $0.08^{\mathrm{eB}}$ & 6.83 & \pm & $0.03^{\mathrm{aA}}$ & 16.80 & \pm & $0.05^{\mathrm{dB}}$ \\
\hline & 12 & 49.10 & \pm & $0.12^{\mathrm{eC}}$ & 6.86 & \pm & $0.00^{\mathrm{aA}}$ & 14.04 & \pm & $0.01^{\mathrm{eC}}$ \\
\hline & 15 & 41.77 & \pm & $0.06^{\mathrm{eD}}$ & 5.95 & \pm & $0.04^{\mathrm{cB}}$ & 10.84 & \pm & $0.03^{\mathrm{eD}}$ \\
\hline & 18 & 40.08 & \pm & $0.06^{\mathrm{eE}}$ & 5.34 & \pm & $0.00^{\mathrm{dC}}$ & 9.22 & \pm & $0.02^{\mathrm{eE}}$ \\
\hline & 21 & 37.35 & \pm & $0.18^{\mathrm{eF}}$ & 4.97 & \pm & $0.01^{\mathrm{dD}}$ & 7.90 & \pm & $0.02^{\mathrm{eF}}$ \\
\hline
\end{tabular}

a-eMeans with different superscripts within the same time group are significantly different $(p<0.05)$. A-FMeans with different superscripts within the same size group are significantly different $(p<0.05)$. 1)After cryogenic milling of ginseng samples with different conditions, the samples were classified by their particle sizes. One treatment condition in each size range was then selected, and samples selected by particle size were roasted and pulverized by cryogenic milling.

\subsection{WSI}

Figure 4 presents the WSI of dried ginseng powder with different roasting times. WSI values tended to increase as the particle size became smaller except for treatments involving roasting at over $18 \mathrm{~min}$. This result was consistent with the findings of a previous study that the WSI value of 30.0 $\mu \mathrm{m}$-sized ginseng powder was increased by $17 \%$ when compared with the $1,000-1,250 \mu \mathrm{m}$-sized 
ginseng powder [18]. Ultrafine grinding removed the cellulose barrier in the plant material, resulting in a smaller particle size, increased water holding capacity, and ultimately increased WSI [18,34]. In addition to particle size, roasting time also had a significant effect on WSI. WSI values of all treatments except for $18 \mathrm{~min}$ and $21 \mathrm{~min}$ were higher than those of the control $(p<0.05)$. This result was explained by dextrinization wherein the starch structure was destroyed by high heat [35]. The starch chain split from amylopectin (insoluble starch) into amylose (soluble starch), thus increasing the amount of soluble materials [35]. In addition, another study also reported that glycosidic linkages were broken down at high temperature (approximately $80^{\circ} \mathrm{C}$ ), resulting in the release of glucose, known as soluble dietary fiber [36]. However, in this study, the WSI values were unexpectedly decreased in case of roasting over $18 \mathrm{~min}$. Another study has also reported a similar result, showing that when ginseng was roasted for $30 \mathrm{~min}$ at $200^{\circ} \mathrm{C}$, the soluble solids content increased until $10 \mathrm{~min}$, but decreased rapidly in case of roasting for over $10 \mathrm{~min}$ [15]. The reason of decreased WSI can be attributed to the burning caused by long heat treatment over $18 \mathrm{~min}$ (Figure 2).

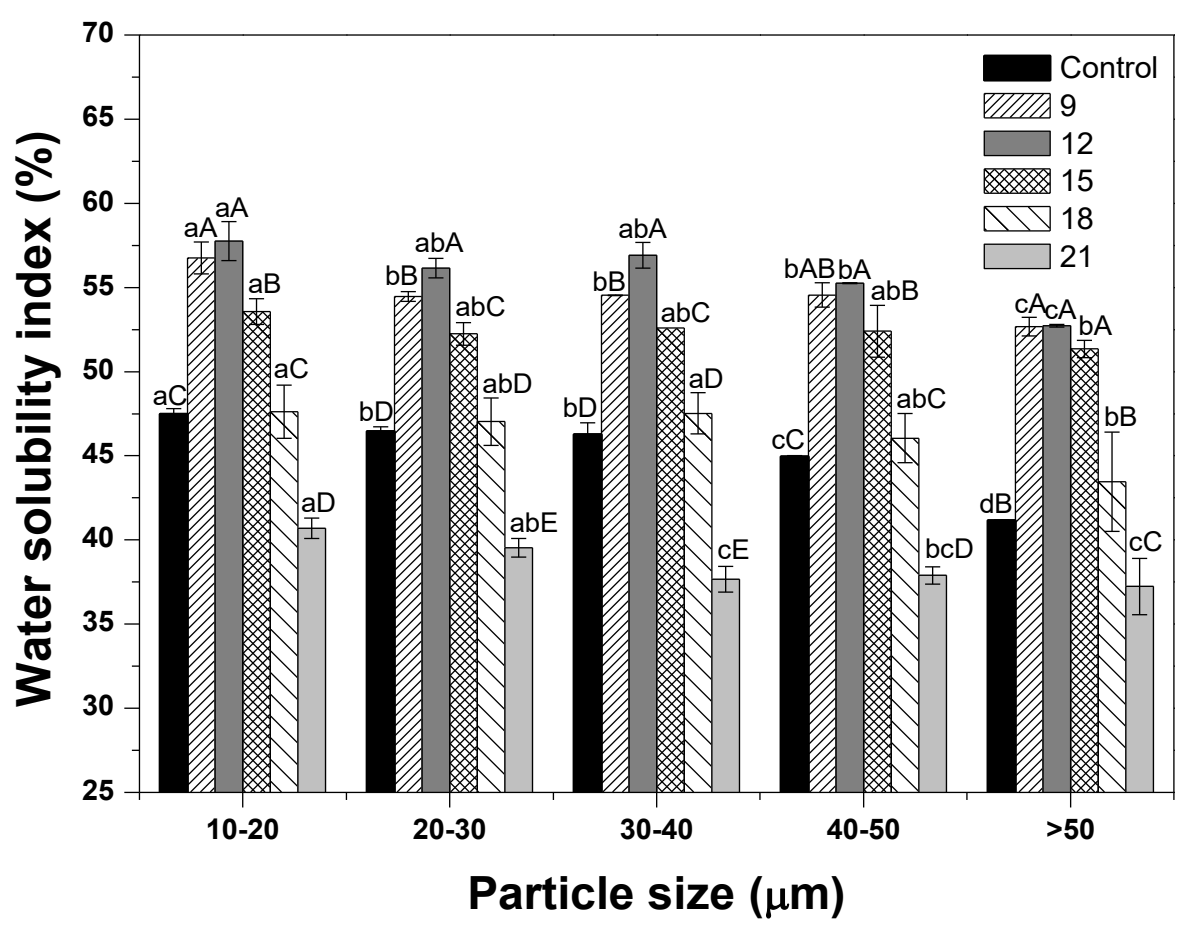

Fig. 4. Water solubility index of dried ginseng powders with different particle sizes according to roasting time. After cryogenic milling of ginseng samples with different conditions, the samples were classified by their particle sizes. One treatment condition in each size range was then selected, and samples selected by particle size were then roasted and pulverized by cryogenic milling. ${ }^{\text {a-d }}$ Means with different superscripts within the same time group are significantly different $(p<0.05)$. A-EMeans with different superscripts within the same size group are significantly different $(p<0.05)$.

\subsection{Antioxidant activity}

The antioxidant activity of ginseng powder is shown in Figure 5. As the particle size decreased, an increasing pattern of antioxidant activity was observed in control samples alone. This result indicated that the ultrafine grinding increased the antioxidant activity by increasing the specific surface area and capillary effect as the particle size decreased (normal grinding, 69.8\%; fine grinding, 70.7\%; and ultrafine grinding, 83.8\%) [37-39]. For the roasting effect, the antioxidant activity values of all roasted samples showed no trend according to particle size, whereas that of roasted samples was significantly higher than that of the control $(p<0.05)$. The roasting process appeared to increase melanoidins by reacting reducing sugars and nitrogen oxides through the Maillard reaction, leading to an increase in antioxidant activity [17]. This result was also concordant with the findings of a study 
by Song et al. (2013) showing that roasting treatment $\left(110^{\circ} \mathrm{C}, 30 \mathrm{~min}\right)$ resulted in 2 times higher antioxidant activity than did the unroasted treatment [40]. Further, antioxidant activity tended to increase with an increase in roasting time except for the ginseng powder sample sized 10-20 $\mu \mathrm{m}$. However, it did not show a significant difference. Seong et al. (2018) reported a result consistent with findings of this study and showed an increasing tendency of antioxidant activity as the roasting time increased [41]. However, no significant difference was observed with roasting for 20 and $30 \mathrm{~min}$ at $170^{\circ} \mathrm{C}(p>0.05)$ [41]. Thus, these results were observed in a narrow range of roasting time in this study.

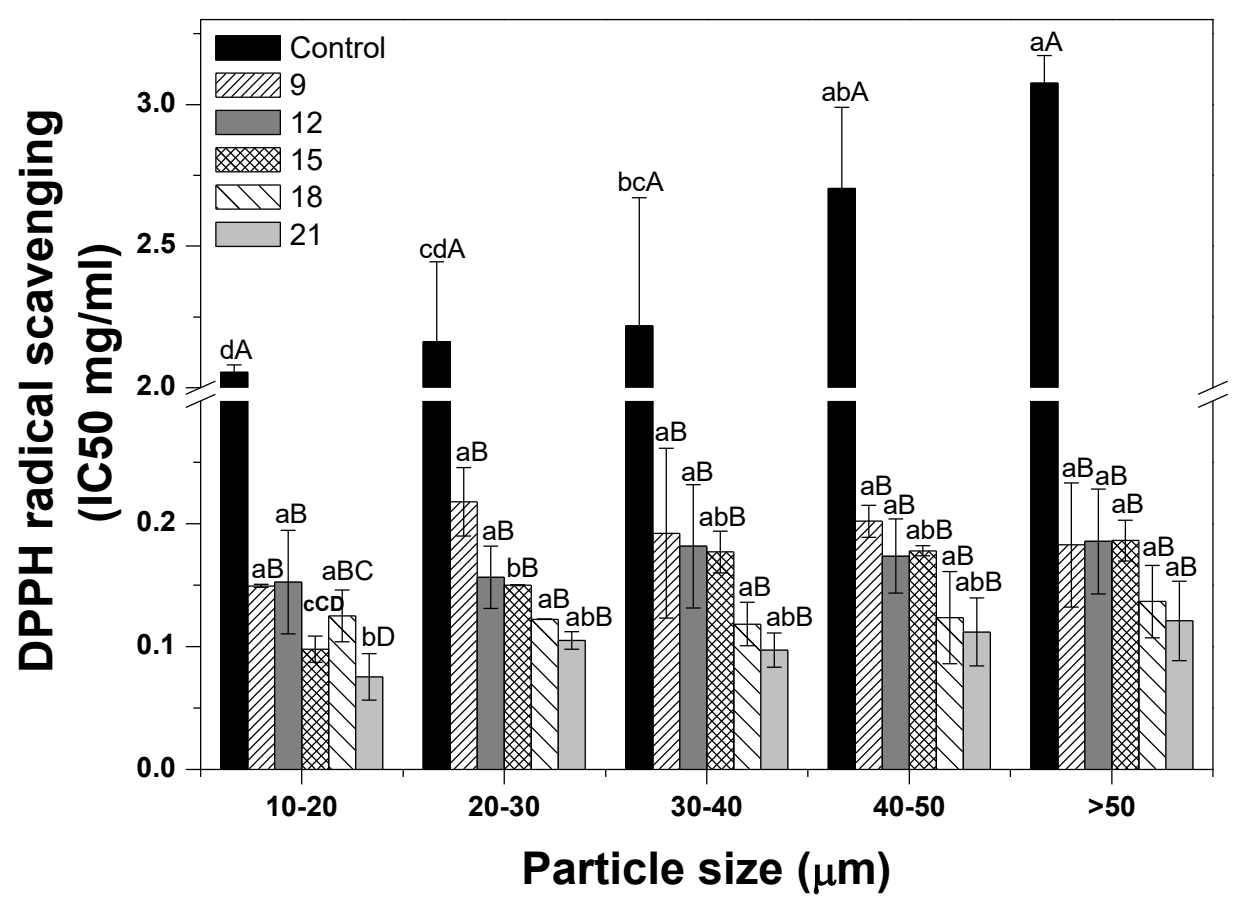

Fig. 5. DPPH radical scavenging activity of dried ginseng powders with different particle sizes according to roasting time. After cryogenic milling of ginseng samples with different conditions, the samples were classified by their particle sizes. One treatment condition in each size range was then selected, and samples selected by particle size were then roasted and pulverized by cryogenic milling. a-cMeans with different superscripts within the same time group are significantly different $(p<0.05)$. A-FMeans with different superscripts within the same size group are significantly different $(p<0.05)$.

\subsection{TPC}

TPC values of ginseng powder were also analyzed (Figure 6). TPC values showed an increasing pattern when compared with control samples. Further, TPC values of roasted ginseng powder with 10-20 $\mu \mathrm{m}$-size tended to increase when compared with those of $>50 \mu \mathrm{m}$ samples. These results were also in agreement with the findings of a previous study that showed that TPCs displayed an increasing pattern as the particle size decreased in five treatments with various particle sizes (M1, $199.17 \mu \mathrm{m} ; \mathrm{M} 2,178.27 \mu \mathrm{m} ; \mathrm{M} 3,85.48 \mu \mathrm{m} ; \mathrm{M} 4.27 .04 \mu \mathrm{m}$; and M5 $20.97 \mu \mathrm{m}$ ) [42]. This phenomenon was explained by the particle size-extraction relationship, indicating that phenolic compounds were easily released due to an increase in the specific surface area as the particle size decreased [37]. According to the roasting treatment, all roasted treatments showed significantly higher TPC values than did the control $(p<0.05)$. TPC values tended to increase as the roasting time increased. Roasting produced a Maillard reaction product with phenol structure and increased TPC values [43]. This can also be explained by the fact that the treatment changes phenolic acids to a free form, which is easier to release from the food matrix owing to cell disruption [44]. These results were similar to those of a 
previous study showing that the TPC values of corn kernels increased as the roasting time $(10,20,30$, 40 , and $50 \mathrm{~min}$ ) increased at $180^{\circ} \mathrm{C}$ [45].

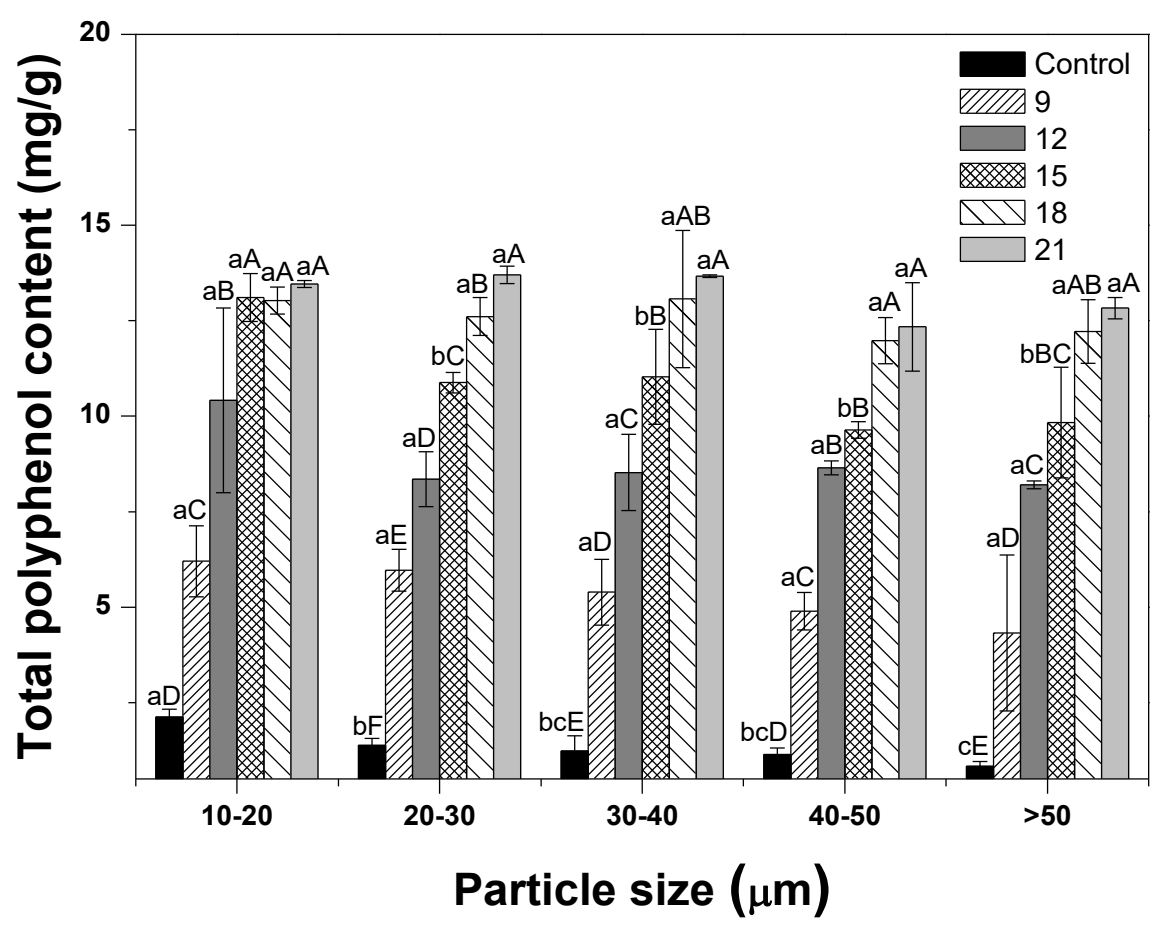

Fig. 6. Total polyphenol content of dried ginseng powders with different particle sizes according to roasting time. After cryogenic milling of ginseng samples with different conditions, the samples were classified by their particle sizes. One treatment condition in each size range was then selected, and samples selected by particle size were then roasted and pulverized by cryogenic milling. ${ }^{\text {a-c Means }}$ with different superscripts within the same time group are significantly different $(p<0.05)$. A-EMeans with different superscripts within the same size group are significantly different $(p<0.05)$.

\subsection{Polysaccharide content}

Figure $7 \mathrm{a}$ and $7 \mathrm{~b}$ present the total and acidic polysaccharide contents, respectively. The total polysaccharide contents of 10-20 $\mu \mathrm{m}$-sized treatments revealed significantly higher values than the $>50 \mu \mathrm{m}$-sized treatments, except for the samples roasted for $21 \mathrm{~min}(p<0.05)$. Cho et al (2010) also reported that ginseng samples with sizes smaller than $150 \mu \mathrm{m}$ had higher total polysaccharide yields comparing to samples with higher sizes [46]. However, the acidic polysaccharide contents did not show an increasing pattern as the particle size decreased. These results were also concordant with a previous study showing that acidic polysaccharides of 10-40 mesh treatment (mean diameter: $>500$ $\mu \mathrm{m}$ ) showed a higher value than those of 40-100 mesh treatment (mean diameter: $162.9 \mu \mathrm{m}$ ) [47]. This phenomenon appeared to be caused by water holding capacity [47]. Water penetration and adsorption are facilitated by structural changes (specific surface increase, ginseng starch damage) arising from decreasing particle size [47]. As a result, the flocculated powder due to an increase of the water holding capacity decreased the diffusion of components from the extractive solvent [47]. For comparison of the roasting treatments, the total polysaccharides of samples sized as $10-20 \mu \mathrm{m}$ showed the highest value when roasting for $12 \mathrm{~min}$, and the other samples with different sizes showed high contents when roasting for $15 \mathrm{~min}$. However, the total polysaccharides of all treatments tended to gradually decrease with roasting for over $18 \mathrm{~min}$ (relatively long heat treatment). In another study, when comparing the light (3-3.5 min), medium (4-5 min), and dark (7-8.5 min) roasting treatments, the total polysaccharide contents of Arabica coffee beans was found to be decreased as the roasting time increased [48]. This was because the long roasting process destroyed or chemically altered the monosaccharides [48]. However, acidic polysaccharides in all roasting treatments showed 
higher values than did the control (not-roasted) despite the absence of any trend according to roasting time $(p<0.05)$. Roasting was considered to decompose the starch components, and facilitate the extraction of acidic polysaccharides [49]. This result suggested that the reduction of total polysaccharides (acidic and neutral) was mainly caused by neutral polysaccharide decomposition [50]. Acidic polysaccharides are known to have higher biological activities than do neutral polysaccharides in ginseng [50], and thus, roasting can be considered an effective pretreatment.
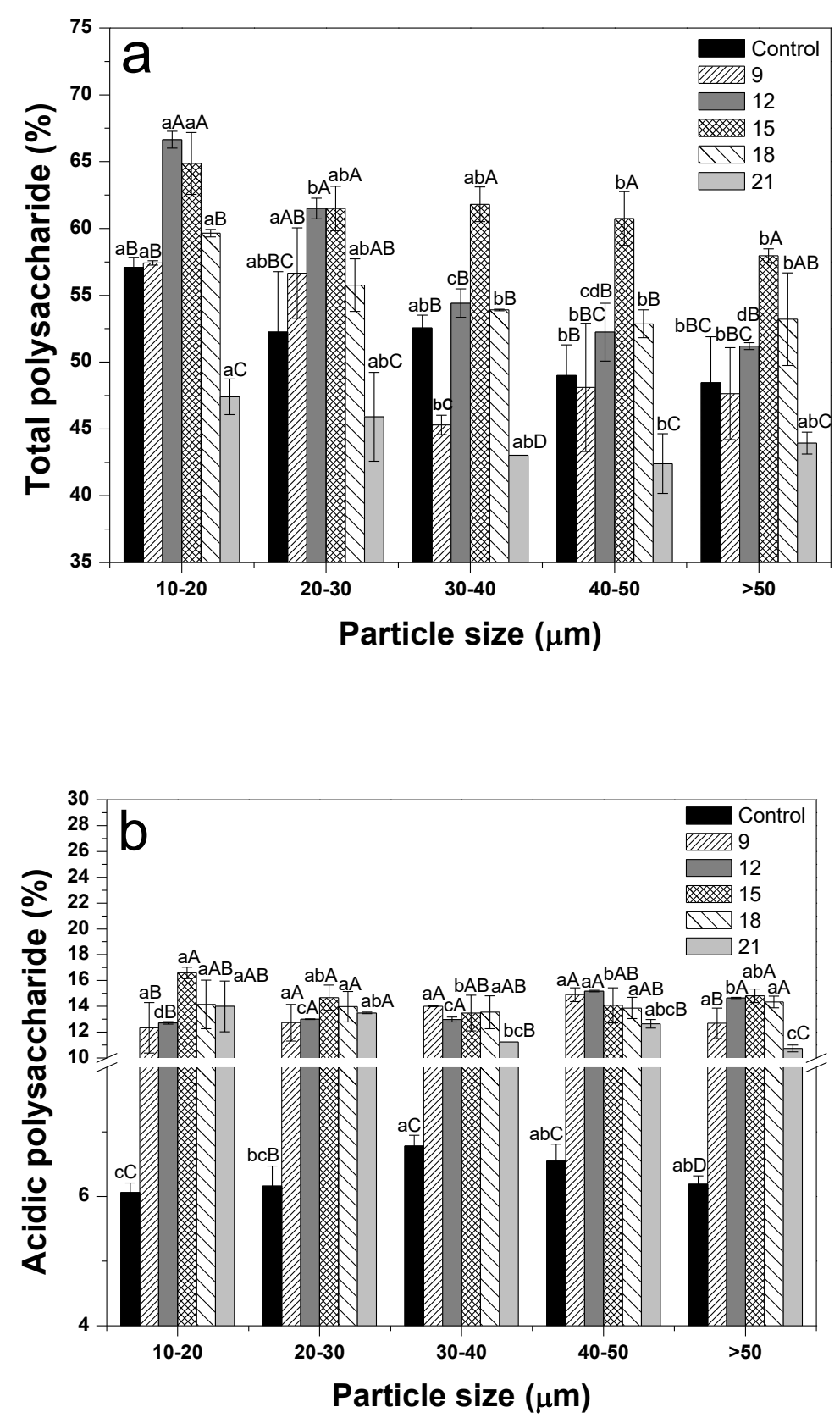

Fig. 7. Polysaccharides of dried ginseng powders with different particle size according to roasting time. After cryogenic milling of ginseng samples with different conditions, the samples were classified by their particle sizes. One treatment condition in each size range was then selected, and samples selected by particle size were then roasted and pulverized by cryogenic milling. ${ }^{a-c}$ Means with different superscripts within the same time group are significantly different $(p<0.05)$. A-EMeans with different superscripts within the same size group are significantly different $(p<0.05)$. 


\subsection{Ginsenoside content}

Ginsenoside content values showed no tendency of changing according to particle size (Table 4). According to the roasting pretreatment, roasted samples with over $20 \mu \mathrm{m}$ size had higher contents than the control except for the samples roasted for $15 \mathrm{~min}$. Seong et al. (2018) reported that the ginsenoside contents of ginseng samples roasted for $20-30 \mathrm{~min}$ at $170^{\circ} \mathrm{C}$ were higher than those in the control (not roasted) [41]. The ginseng samples with 10-30 $\mu \mathrm{m}$ size revealed the highest ginsenoside contents in case of roasting for $18 \mathrm{~min}$. However, samples with over $30 \mu \mathrm{m}$ size indicated the highest contents when roasting for $12 \mathrm{~min}(p<0.05)$. These results indicated that the roasting time to obtain the optimum ginsenoside content varied according to particle size.

$\mathrm{Rg} 1, \mathrm{Re}$, and $\mathrm{Rb} 1$, known as the main components of ginseng, also indicated a pattern according to roasting time. $\mathrm{Rg} 1$ in all size treatments showed the highest contents with roasting for $9 \min (p<$ 0.05). Re showed the highest content with roasting for 12 min except for samples with 10-20 $\mu \mathrm{m}$ size. Finally, $\mathrm{Rb} 1$ of the 10-30 $\mu \mathrm{m}$-sized samples showed the highest contents with roasting for $18 \mathrm{~min}$, whereas samples with over $30 \mu \mathrm{m}$ size and relatively larger size showed the highest contents with roasting for $12 \mathrm{~min}(p<0.05)$. Therefore, the ginsenoside content was observed to change with particle size and roasting time.

Table 4. Ginsenosides content of dried ginseng powder with different roasting time conditions

\begin{tabular}{|c|c|c|c|c|c|c|c|c|c|}
\hline \multirow[b]{2}{*}{$\begin{array}{l}\text { Size } \\
(\mu \mathrm{m})\end{array}$} & \multirow{2}{*}{$\begin{array}{l}\text { Roasting1) } \\
\begin{array}{l}\text { Time } \\
(\mathrm{min})\end{array} \\
\end{array}$} & \multicolumn{8}{|c|}{ Ginsenosides content $(\mathrm{mg} / \mathrm{g})$} \\
\hline & & $\operatorname{Rg} 1$ & $\operatorname{Re}$ & $\mathbf{R f}$ & Rb1 & Rc & Rb2 & Rb3 & Rd \\
\hline \multirow{13}{*}{$\begin{array}{l}10- \\
20\end{array}$} & Control & $3.68 \pm 0.03 \mathrm{C}$ & $3.41 \pm 0.02^{\mathrm{D}}$ & $1.46 \pm 0.08^{\mathrm{B}}$ & $4.26 \pm 0.03 \mathrm{C}$ & $1.14 \pm 0.04^{\mathrm{C}}$ & $0.97 \pm 0.03 \mathrm{C}$ & $0.19 \pm 0.02^{\mathrm{C}}$ & $0.31 \pm 0.01^{\mathrm{C}}$ \\
\hline & 9 & $4.62 \pm 0.02^{\mathrm{A}}$ & $2.82 \pm 0.01^{\mathrm{E}}$ & $1.01 \pm 0.01^{\mathrm{C}}$ & $4.18 \pm 0.04^{\mathrm{C}}$ & $1.01 \pm 0.03^{\mathrm{D}}$ & $0.91 \pm 0.01^{\mathrm{C}}$ & $0.15 \pm 0.00^{\mathrm{C}}$ & $0.35 \pm 0.00^{\mathrm{C}}$ \\
\hline & 12 & $3.91 \pm 0.13^{\text {в }}$ & $7.56 \pm 0.21^{\mathrm{B}}$ & $1.77 \pm 0.00^{\mathrm{A}}$ & $8.34 \pm 0.30^{\text {в }}$ & $3.35 \pm 0.13^{\mathrm{B}}$ & $2.99 \pm 0.11^{\mathrm{B}}$ & $0.51 \pm 0.02^{\mathrm{B}}$ & $0.91 \pm 0.03^{\mathrm{B}}$ \\
\hline & 15 & $2.30 \pm 0.14^{\mathrm{E}}$ & $1.84 \pm 0.10^{\mathrm{F}}$ & $0.73 \pm 0.01^{\mathrm{D}}$ & $3.04 \pm 0.03^{\mathrm{D}}$ & $1.05 \pm 0.03^{\mathrm{CD}}$ & $0.92 \pm 0.01^{\mathrm{C}}$ & $0.17 \pm 0.01^{\mathrm{C}}$ & $0.18 \pm 0.01^{\mathrm{D}}$ \\
\hline & 18 & $4.00 \pm 0.09 \mathrm{~B}$ & $8.01 \pm 0.17^{\mathrm{A}}$ & $1.85 \pm 0.04^{\mathrm{A}}$ & $9.88 \pm 0.82^{\mathrm{A}}$ & $4.07 \pm 0.10^{\mathrm{A}}$ & $3.60 \pm 0.11^{\mathrm{A}}$ & $0.66 \pm 0.03^{\mathrm{A}}$ & $1.02 \pm 0.05^{\mathrm{A}}$ \\
\hline & 21 & $3.35 \pm 0.05^{\mathrm{D}}$ & $6.88 \pm 0.14^{\mathrm{C}}$ & $0.27 \pm 0.08^{\mathrm{E}}$ & $\mathrm{ND}^{\mathrm{E}}$ & $\mathrm{ND}^{\mathrm{E}}$ & $\mathrm{ND}^{\mathrm{D}}$ & $\mathrm{ND}^{\mathrm{D}}$ & $0.21 \pm 0.02^{\mathrm{D}}$ \\
\hline & $\begin{array}{l}\text { Time } \\
(\mathrm{min})\end{array}$ & $\operatorname{Rg} 2(S)$ & Rg2(R) & $\operatorname{Rg} 3(S)$ & $\operatorname{Rg} 3(\mathrm{R})$ & Rh1(S) & $\mathrm{Rh} 2(\mathrm{~S})$ & Sum & \\
\hline & Control & $0.17 \pm 0.01^{\mathrm{D}}$ & $0.09 \pm 0.01^{\mathrm{C}}$ & $\mathrm{ND}^{\mathrm{B}}$ & $\mathrm{ND}^{\mathrm{B}}$ & $0.10 \pm 0.02^{\mathrm{B}}$ & ND & $15.77 \pm 0.02^{\mathrm{bc}}$ & \\
\hline & 9 & $0.15 \pm 0.01^{\mathrm{D}}$ & $0.14 \pm 0.00^{\mathrm{BC}}$ & $\mathrm{ND}^{\mathrm{B}}$ & $\mathrm{ND}^{\mathrm{B}}$ & $0.03 \pm 0.00^{\mathrm{D}}$ & ND & $15.39 \pm 0.09 \mathrm{bc}$ & \\
\hline & 12 & $0.73 \pm 0.02^{\mathrm{B}}$ & $0.48 \pm 0.02^{\mathrm{A}}$ & $\mathrm{ND}^{\mathrm{B}}$ & $\mathrm{ND}^{\mathrm{B}}$ & $0.04 \pm 0.00^{\mathrm{D}}$ & ND & $30.64 \pm 1.03^{\text {св }}$ & \\
\hline & 15 & $0.26 \pm 0.04^{C}$ & $0.10 \pm 0.04 \mathrm{C}$ & $0.19 \pm 0.01^{\mathrm{A}}$ & $0.07 \pm 0.03^{\mathrm{A}}$ & $0.22 \pm 0.00^{\mathrm{A}}$ & ND & $11.06 \pm 0.03^{\mathrm{cD}}$ & \\
\hline & 18 & $0.89 \pm 0.02^{\mathrm{A}}$ & $0.34 \pm 0.30^{\mathrm{AB}}$ & $\mathrm{ND}^{\mathrm{B}}$ & $\mathrm{ND}^{\mathrm{B}}$ & $0.07 \pm 0.00^{\mathrm{C}}$ & ND & $34.39 \pm 1.10^{\mathrm{aA}}$ & \\
\hline & 21 & $\mathrm{ND}^{\mathrm{E}}$ & $0.19 \pm 0.04^{\mathrm{BC}}$ & $\mathrm{ND}^{\mathrm{B}}$ & $\mathrm{ND}^{\mathrm{B}}$ & $\mathrm{ND}^{\mathrm{E}}$ & ND & $10.73 \pm 0.00^{\mathrm{dD}}$ & \\
\hline $\begin{array}{l}\text { Size } \\
(\mu \mathrm{m})\end{array}$ & $\begin{array}{l}\text { Time } \\
(\mathrm{min})\end{array}$ & Rg1 & $\operatorname{Re}$ & $R f$ & Rb1 & Rc & $\mathrm{Rb} 2$ & Rb3 & Rd \\
\hline \multirow{5}{*}{$\begin{array}{l}20- \\
30\end{array}$} & Control & $3.28 \pm 0.04^{\mathrm{B}}$ & $2.68 \pm 0.02^{\mathrm{C}}$ & $0.81 \pm 0.70^{\mathrm{B}}$ & $3.60 \pm 0.06^{\mathrm{D}}$ & $0.90 \pm 0.02^{\mathrm{D}}$ & $0.78 \pm 0.02^{\mathrm{D}}$ & $0.19 \pm 0.01^{\mathrm{C}}$ & $0.28 \pm 0.02^{\mathrm{D}}$ \\
\hline & 9 & $4.59 \pm 0.07^{\mathrm{A}}$ & $2.37 \pm 0.03^{\mathrm{CD}}$ & $0.96 \pm 0.03^{\text {в }}$ & $4.24 \pm 0.06^{\mathrm{C}}$ & $1.12 \pm 0.01^{\mathrm{C}}$ & $1.03 \pm 0.01^{\mathrm{D}}$ & $0.17 \pm 0.00^{C}$ & $0.38 \pm 0.01^{\mathrm{C}}$ \\
\hline & 12 & $3.10 \pm 0.05^{\mathrm{B}}$ & $8.88 \pm 0.15^{\mathrm{A}}$ & $1.65 \pm 0.06^{\mathrm{A}}$ & $9.62 \pm 0.15^{\mathrm{A}}$ & $4.14 \pm 0.06^{\mathrm{A}}$ & $3.64 \pm 0.07^{\mathrm{A}}$ & $0.62 \pm 0.01^{\mathrm{A}}$ & $1.14 \pm 0.01^{\mathrm{A}}$ \\
\hline & 15 & $2.17 \pm 0.07 \mathrm{C}$ & $2.08 \pm 0.08^{\mathrm{D}}$ & $0.73 \pm 0.03^{\text {в }}$ & $3.08 \pm 0.16^{\mathrm{E}}$ & $1.05 \pm 0.00^{\mathrm{C}}$ & $0.84 \pm 0.02^{\mathrm{D}}$ & $0.14 \pm 0.01^{\mathrm{C}}$ & $0.23 \pm 0.02^{\mathrm{E}}$ \\
\hline & 18 & $2.98 \pm 0.37^{\mathrm{B}}$ & $8.71 \pm 0.24^{\mathrm{A}}$ & $1.67 \pm 0.17^{\mathrm{A}}$ & $9.71 \pm 0.34^{\mathrm{A}}$ & $4.09 \pm 0.10^{\mathrm{A}}$ & $3.29 \pm 0.41^{\mathrm{B}}$ & $0.60 \pm 0.09^{A}$ & $1.11 \pm 0.02^{\mathrm{A}}$ \\
\hline
\end{tabular}




\begin{tabular}{|c|c|c|c|c|c|c|c|c|c|}
\hline & 21 & $2.11 \pm 0.03^{C}$ & $5.47 \pm 0.35^{\mathrm{B}}$ & $1.60 \pm 0.10 \mathrm{~A}$ & $6.49 \pm 0.04 \mathrm{~B}$ & $3.14 \pm 0.01^{\mathrm{B}}$ & $2.88 \pm 0.00^{\mathrm{C}}$ & $0.51 \pm 0.02^{\mathrm{B}}$ & $0.87 \pm 0.05^{\mathrm{B}}$ \\
\hline & $\begin{array}{l}\text { Time } \\
\text { (min) }\end{array}$ & Rg2(S) & Rg2(R) & $\operatorname{Rg} 3(S)$ & $\operatorname{Rg} 3(\mathrm{R})$ & $\operatorname{Rh1}(S)$ & $\mathrm{Rh} 2(\mathrm{~S})$ & Sum & \\
\hline & Control & $0.06 \pm 0.02^{\mathrm{E}}$ & $0.06 \pm 0.01^{\mathrm{E}}$ & $\mathrm{ND}^{\mathrm{C}}$ & $\mathrm{ND}^{\mathrm{B}}$ & $0.10 \pm 0.01^{\mathrm{C}}$ & ND & $12.75 \pm 0.07 \mathrm{cD}$ & \\
\hline & 9 & $0.15 \pm 0.00^{\mathrm{D}}$ & $0.14 \pm 0.00^{\mathrm{D}}$ & $\mathrm{NDC}^{\mathrm{C}}$ & $\mathrm{ND}^{\mathrm{B}}$ & $0.04 \pm 0.00^{\mathrm{E}}$ & ND & $15.18 \pm 0.20^{\mathrm{bC}}$ & \\
\hline & 12 & $0.93 \pm 0.01^{\mathrm{B}}$ & $0.63 \pm 0.01^{\mathrm{A}}$ & $\mathrm{ND}^{\mathrm{C}}$ & $\mathrm{ND}^{\mathrm{B}}$ & $0.04 \pm 0.00^{\mathrm{E}}$ & ND & $34.39 \pm 0.55^{\mathrm{bA}}$ & \\
\hline & 15 & $0.32 \pm 0.02^{\mathrm{C}}$ & $0.16 \pm 0.01^{\mathrm{D}}$ & $0.19 \pm 0.02^{\mathrm{B}}$ & $0.05 \pm 0.01^{\mathrm{A}}$ & $0.22 \pm 0.03^{в}$ & ND & $11.26 \pm 0.03^{\mathrm{bcE}}$ & \\
\hline & 18 & $0.97 \pm 0.02^{\mathrm{B}}$ & $0.56 \pm 0.07^{в}$ & $\mathrm{ND}^{\mathrm{C}}$ & $\mathrm{ND}^{\mathrm{B}}$ & $0.07 \pm 0.01^{\mathrm{D}}$ & ND & $34.41 \pm 0.97 \mathrm{aA}$ & \\
\hline & 21 & $1.21 \pm 0.08^{\mathrm{A}}$ & $0.39 \pm 0.03^{\mathrm{C}}$ & $0.46 \pm 0.03^{\mathrm{A}}$ & $\mathrm{ND}^{\mathrm{B}}$ & $0.26 \pm 0.02^{\mathrm{A}}$ & ND & $25.80 \pm 0.15^{\mathrm{aв}}$ & \\
\hline $\begin{array}{l}\text { Size } \\
(\mu \mathrm{m})\end{array}$ & $\begin{array}{l}\text { Time } \\
\text { (min) }\end{array}$ & Rg1 & $\operatorname{Re}$ & $R f$ & Rb1 & Rc & $\mathrm{Rb} 2$ & Rb3 & $\mathrm{Rd}$ \\
\hline \multirow{13}{*}{$\begin{array}{l}30- \\
40\end{array}$} & Control & $3.93 \pm 0.08^{\mathrm{B}}$ & $3.24 \pm 0.04^{\mathrm{D}}$ & $1.13 \pm 0.31^{\mathrm{C}}$ & $4.60 \pm 0.28^{\mathrm{D}}$ & $1.57 \pm 0.16^{\mathrm{D}}$ & $1.33 \pm 0.17 \mathrm{C}$ & $0.19 \pm 0.04^{\mathrm{D}}$ & $0.42 \pm 0.07^{\mathrm{E}}$ \\
\hline & 9 & $5.20 \pm 0.08^{\mathrm{A}}$ & $2.94 \pm 0.03^{\mathrm{E}}$ & $1.04 \pm 0.01^{\mathrm{C}}$ & $5.16 \pm 0.09 \mathrm{C}$ & $1.42 \pm 0.03^{\mathrm{D}}$ & $1.29 \pm 0.04^{C}$ & $0.21 \pm 0.01^{\mathrm{D}}$ & $0.49 \pm 0.01^{\mathrm{D}}$ \\
\hline & 12 & $3.20 \pm 0.02^{\mathrm{C}}$ & $9.94 \pm 0.03^{\mathrm{A}}$ & $1.75 \pm 0.07^{\mathrm{A}}$ & $11.14 \pm 0.02^{\mathrm{A}}$ & $4.54 \pm 0.06^{\mathrm{A}}$ & $4.00 \pm 0.04^{\mathrm{A}}$ & $0.68 \pm 0.01^{\mathrm{B}}$ & $1.30 \pm 0.01^{\mathrm{A}}$ \\
\hline & 15 & $2.14 \pm 0.05^{\mathrm{F}}$ & $2.26 \pm 0.07 \mathrm{~F}$ & $0.68 \pm 0.00^{\mathrm{D}}$ & $3.39 \pm 0.07 \mathrm{E}$ & $1.26 \pm 0.09^{\mathrm{E}}$ & $1.06 \pm 0.06^{\mathrm{D}}$ & $0.15 \pm 0.04^{\mathrm{E}}$ & $0.26 \pm 0.03^{F}$ \\
\hline & 18 & $3.02 \pm 0.10^{\mathrm{D}}$ & $8.59 \pm 0.22^{\mathrm{B}}$ & $1.81 \pm 0.06^{\mathrm{A}}$ & $10.01 \pm 0.37^{\mathrm{B}}$ & $4.31 \pm 0.13^{\mathrm{B}}$ & $4.03 \pm 0.11^{\mathrm{A}}$ & $0.74 \pm 0.03^{\mathrm{A}}$ & $1.14 \pm 0.02^{\mathrm{B}}$ \\
\hline & 21 & $1.79 \pm 0.02^{\mathrm{E}}$ & $4.70 \pm 0.08^{\mathrm{C}}$ & $1.51 \pm 0.03^{\mathrm{B}}$ & $5.40 \pm 0.06^{\mathrm{C}}$ & $2.53 \pm 0.05^{\mathrm{C}}$ & $2.29 \pm 0.03^{\mathrm{B}}$ & $0.43 \pm 0.00^{\mathrm{C}}$ & $0.74 \pm 0.02^{\mathrm{C}}$ \\
\hline & $\begin{array}{l}\text { Time } \\
(\mathrm{min})\end{array}$ & Rg2(S) & Rg2(R) & $\operatorname{Rg} 3(S)$ & Rg3(R) & Rh1(S) & $\mathrm{Rh} 2(\mathrm{~S})$ & Sum & \\
\hline & Control & $0.20 \pm 0.08^{\mathrm{E}}$ & $0.11 \pm 0.12^{\mathrm{E}}$ & $\mathrm{ND}^{\mathrm{C}}$ & $\mathrm{ND}^{\mathrm{B}}$ & $0.05 \pm 0.09 \mathrm{C}$ & ND & $16.77 \pm 0.10^{\mathrm{aE}}$ & \\
\hline & 9 & $0.20 \pm 0.00^{\mathrm{E}}$ & $0.20 \pm 0.00^{\mathrm{D}}$ & $\mathrm{ND}^{\mathrm{C}}$ & $\mathrm{ND}^{\mathrm{B}}$ & $0.05 \pm 0.00^{\mathrm{C}}$ & ND & $18.20 \pm 0.25^{\mathrm{aD}}$ & \\
\hline & 12 & $1.04 \pm 0.01^{\mathrm{C}}$ & $0.73 \pm 0.01^{\mathrm{A}}$ & $\mathrm{ND}^{\mathrm{C}}$ & $\mathrm{ND}^{\mathrm{B}}$ & $0.05 \pm 0.00^{C}$ & ND & $38.36 \pm 0.21^{\mathrm{aA}}$ & \\
\hline & 15 & $0.32 \pm 0.01^{\mathrm{D}}$ & $0.09 \pm 0.00^{\mathrm{E}}$ & $0.18 \pm 0.00^{\mathrm{B}}$ & $0.05 \pm 0.00^{\mathrm{A}}$ & $0.20 \pm 0.04^{\mathrm{B}}$ & ND & $12.05 \pm 0.03^{\mathrm{aF}}$ & \\
\hline & 18 & $1.13 \pm 0.04^{\mathrm{B}}$ & $0.56 \pm 0.01^{\mathrm{B}}$ & $0.02 \pm 0.01^{\mathrm{C}}$ & $\mathrm{ND}^{\mathrm{B}}$ & $0.10 \pm 0.01^{\mathrm{C}}$ & ND & $35.46 \pm 1.01^{\mathrm{aB}}$ & \\
\hline & 21 & $1.28 \pm 0.03^{\mathrm{A}}$ & $0.38 \pm 0.01^{\mathrm{C}}$ & $0.67 \pm 0.01^{\mathrm{A}}$ & $\mathrm{ND}^{\mathrm{B}}$ & $0.33 \pm 0.01^{\mathrm{A}}$ & ND & $22.05 \pm 0.31^{\mathrm{cC}}$ & \\
\hline $\begin{array}{l}\text { Size } \\
(\mu \mathrm{m})\end{array}$ & $\begin{array}{l}\text { Time } \\
(\mathrm{min})\end{array}$ & Rg1 & $\operatorname{Re}$ & $\mathrm{Rf}$ & $\mathbf{R b} 1$ & Rc & $\mathbf{R b} 2$ & Rb3 & $\mathrm{Rd}$ \\
\hline \multirow{10}{*}{$\begin{array}{l}40- \\
50\end{array}$} & Control & $3.14 \pm 0.02^{\mathrm{C}}$ & $2.70 \pm 0.01^{\mathrm{E}}$ & $1.05 \pm 0.03^{\mathrm{C}}$ & $3.23 \pm 0.14^{\mathrm{E}}$ & $0.73 \pm 0.06^{\mathrm{E}}$ & $0.66 \pm 0.04^{\mathrm{F}}$ & $0.17 \pm 0.01^{\mathrm{C}}$ & $0.25 \pm 0.02^{\mathrm{E}}$ \\
\hline & 9 & $4.93 \pm 0.03^{\mathrm{A}}$ & $3.41 \pm 0.05^{\mathrm{D}}$ & $1.08 \pm 0.01^{\mathrm{C}}$ & $5.38 \pm 0.06^{\mathrm{D}}$ & $1.35 \pm 0.02^{\mathrm{C}}$ & $1.20 \pm 0.05^{\mathrm{D}}$ & $0.19 \pm 0.01^{\mathrm{C}}$ & $0.49 \pm 0.01^{\mathrm{D}}$ \\
\hline & 12 & $3.67 \pm 0.06^{\mathrm{B}}$ & $8.68 \pm 0.11^{\mathrm{A}}$ & $1.61 \pm 0.04^{\mathrm{B}}$ & $10.22 \pm 0.14^{\mathrm{A}}$ & $4.01 \pm 0.05^{\mathrm{A}}$ & $3.55 \pm 0.06^{\mathrm{A}}$ & $0.58 \pm 0.00^{\mathrm{A}}$ & $1.23 \pm 0.04^{\mathrm{A}}$ \\
\hline & 15 & $2.16 \pm 0.03^{F}$ & $1.85 \pm 0.01^{\mathrm{F}}$ & $0.76 \pm 0.01^{\mathrm{D}}$ & $2.95 \pm 0.05^{\mathrm{F}}$ & $1.04 \pm 0.06^{\mathrm{D}}$ & $0.91 \pm 0.03^{\mathrm{E}}$ & $0.18 \pm 0.07 \mathrm{C}$ & $0.19 \pm 0.01^{\mathrm{F}}$ \\
\hline & 18 & $2.94 \pm 0.02^{\mathrm{D}}$ & $8.46 \pm 0.06^{\mathrm{B}}$ & $1.78 \pm 0.02^{\mathrm{A}}$ & $9.53 \pm 0.16^{\mathrm{B}}$ & $4.03 \pm 0.02^{\mathrm{A}}$ & $3.44 \pm 0.03^{\mathrm{B}}$ & $0.62 \pm 0.01^{\mathrm{A}}$ & $1.08 \pm 0.02^{\mathrm{B}}$ \\
\hline & 21 & $2.36 \pm 0.03^{\mathrm{E}}$ & $5.67 \pm 0.06^{\mathrm{C}}$ & $1.64 \pm 0.02^{\mathrm{B}}$ & $6.93 \pm 0.06^{\mathrm{C}}$ & $2.63 \pm 0.02^{\mathrm{B}}$ & $2.29 \pm 0.06^{\mathrm{C}}$ & $0.45 \pm 0.01^{\mathrm{B}}$ & $0.72 \pm 0.03^{C}$ \\
\hline & $\begin{array}{l}\text { Time } \\
(\mathrm{min})\end{array}$ & Rg2(S) & Rg2(R) & $\operatorname{Rg} 3(S)$ & Rg3(R) & $\operatorname{Rh1}(S)$ & $\mathrm{Rh} 2(\mathrm{~S})$ & Sum & \\
\hline & Control & $0.07 \pm 0.01^{\mathrm{E}}$ & $0.02 \pm 0.01^{\mathrm{E}}$ & $\mathrm{ND}^{\mathrm{D}}$ & $\mathrm{ND}^{\mathrm{B}}$ & $0.11 \pm 0.01^{\mathrm{B}}$ & ND & $12.13 \pm 0.03 \mathrm{cE}$ & \\
\hline & 9 & $0.21 \pm 0.00^{\mathrm{D}}$ & $0.25 \pm 0.00^{\mathrm{C}}$ & NDD & $\mathrm{ND}^{\mathrm{B}}$ & $0.05 \pm 0.00^{C}$ & ND & $18.52 \pm 0.22^{\mathrm{aD}}$ & \\
\hline & 12 & $0.86 \pm 0.01^{\mathrm{C}}$ & $0.67 \pm 0.01^{\mathrm{A}}$ & NDD & $\mathrm{ND}^{\mathrm{B}}$ & $0.05 \pm 0.00^{C}$ & ND & $35.14 \pm 0.50^{\mathrm{bA}}$ & \\
\hline
\end{tabular}




\begin{tabular}{|c|c|c|c|c|c|c|c|c|c|}
\hline & 15 & $0.27 \pm 0.08^{\mathrm{D}}$ & $0.16 \pm 0.04^{\mathrm{D}}$ & $0.19 \pm 0.02^{\mathrm{B}}$ & $0.05 \pm 0.05^{\mathrm{A}}$ & $0.23 \pm 0.06^{\mathrm{A}}$ & ND & $10.93 \pm 0.04^{\mathrm{cF}^{\mathrm{F}}}$ & \\
\hline & 18 & $1.12 \pm 0.03^{\mathrm{B}}$ & $0.65 \pm 0.01^{\mathrm{A}}$ & $0.03 \pm 0.00^{C}$ & $\mathrm{ND}^{\mathrm{B}}$ & $0.12 \pm 0.00^{\mathrm{B}}$ & ND & $33.73 \pm 0.42^{\mathrm{aB}}$ & \\
\hline & 21 & $1.19 \pm 0.01^{\mathrm{A}}$ & $0.47 \pm 0.01^{\mathrm{B}}$ & $0.38 \pm 0.01^{\mathrm{A}}$ & $\mathrm{ND}^{\mathrm{B}}$ & $0.25 \pm 0.00^{\mathrm{A}}$ & ND & $24.99 \pm 0.28^{\mathrm{bC}}$ & \\
\hline $\begin{array}{l}\text { Size } \\
(\mu \mathrm{m})\end{array}$ & $\begin{array}{l}\text { Time } \\
\text { (min) }\end{array}$ & Rg1 & $\operatorname{Re}$ & $R f$ & Rb1 & Rc & $\mathrm{Rb} 2$ & Rb3 & $\mathrm{Rd}$ \\
\hline \multirow{13}{*}{$>50$} & Control & $3.81 \pm 0.10^{\mathrm{B}}$ & $3.27 \pm 0.05^{\mathrm{D}}$ & $1.50 \pm 0.05^{\mathrm{B}}$ & $4.20 \pm 0.11^{\mathrm{E}}$ & $1.10 \pm 0.05^{\mathrm{E}}$ & $0.94 \pm 0.05^{\mathrm{E}}$ & $0.20 \pm 0.03^{C}$ & $0.29 \pm 0.03^{E}$ \\
\hline & 9 & $4.47 \pm 0.16^{\mathrm{A}}$ & $3.53 \pm 0.08^{\mathrm{D}}$ & $1.13 \pm 0.04^{\mathrm{C}}$ & $5.33 \pm 0.19 \mathrm{D}$ & $1.53 \pm 0.06^{\mathrm{D}}$ & $1.36 \pm 0.05^{\mathrm{D}}$ & $0.24 \pm 0.01^{\mathrm{C}}$ & $0.47 \pm 0.01^{\mathrm{D}}$ \\
\hline & 12 & $2.91 \pm 0.07 \mathrm{C}$ & $8.32 \pm 0.32^{\mathrm{A}}$ & $1.52 \pm 0.03^{\text {В }}$ & $9.65 \pm 0.33^{\mathrm{A}}$ & $4.15 \pm 0.18^{\mathrm{A}}$ & $3.77 \pm 0.15^{\mathrm{A}}$ & $0.62 \pm 0.03^{\mathrm{A}}$ & $1.18 \pm 0.07^{\mathrm{A}}$ \\
\hline & 15 & $2.22 \pm 0.01^{\mathrm{E}}$ & $1.98 \pm 0.05^{\mathrm{E}}$ & $0.81 \pm 0.00^{\mathrm{D}}$ & $3.14 \pm 0.03^{F}$ & $1.14 \pm 0.07 \mathrm{E}$ & $0.96 \pm 0.03^{\mathrm{E}}$ & $0.20 \pm 0.06^{\mathrm{C}}$ & $0.27 \pm 0.03^{E}$ \\
\hline & 18 & $2.74 \pm 0.03^{\mathrm{D}}$ & $7.55 \pm 0.09^{в}$ & $1.66 \pm 0.02^{\mathrm{A}}$ & $8.91 \pm 0.05^{\mathrm{B}}$ & $3.73 \pm 0.02^{\mathrm{B}}$ & $3.27 \pm 0.03^{\text {в }}$ & $0.62 \pm 0.01^{\mathrm{A}}$ & $0.97 \pm 0.02^{\mathrm{B}}$ \\
\hline & 21 & $1.96 \pm 0.06^{\mathrm{F}}$ & $4.76 \pm 0.22^{C}$ & $1.50 \pm 0.03^{\mathrm{B}}$ & $5.86 \pm 0.17 \mathrm{C}$ & $2.48 \pm 0.06^{\mathrm{C}}$ & $2.22 \pm 0.05^{\mathrm{C}}$ & $0.42 \pm 0.00^{\mathrm{B}}$ & $0.72 \pm 0.02^{\mathrm{C}}$ \\
\hline & $\begin{array}{l}\text { Time } \\
(\mathrm{min})\end{array}$ & Rg2(S) & Rg2(R) & Rg3(S) & $\operatorname{Rg} 3(\mathrm{R})$ & Rh1(S) & $\mathrm{Rh} 2(\mathrm{~S})$ & Sum & \\
\hline & Control & $0.16 \pm 0.03 \mathrm{E}$ & $0.09 \pm 0.01^{\mathrm{F}}$ & $\mathrm{ND}^{\mathrm{D}}$ & $\mathrm{ND}^{\mathrm{D}}$ & $0.11 \pm 0.02^{\mathrm{C}}$ & ND & $15.66 \pm 0.04^{\mathrm{bE}}$ & \\
\hline & 9 & $0.29 \pm 0.02^{\mathrm{D}}$ & $0.25 \pm 0.01^{\mathrm{D}}$ & $\mathrm{ND}^{\mathrm{D}}$ & $\mathrm{ND}^{\mathrm{D}}$ & $0.05 \pm 0.00^{\mathrm{D}}$ & ND & $18.66 \pm 0.62^{\mathrm{aD}}$ & \\
\hline & 12 & $0.91 \pm 0.03^{C}$ & $0.55 \pm 0.01^{\mathrm{B}}$ & $0.01 \pm 0.02^{\mathrm{D}}$ & $\mathrm{ND}^{\mathrm{D}}$ & $0.04 \pm 0.00^{\mathrm{D}}$ & ND & $33.64 \pm 1.22^{\mathrm{bA}}$ & \\
\hline & 15 & $0.33 \pm 0.04^{\mathrm{D}}$ & $0.17 \pm 0.02^{\mathrm{E}}$ & $0.26 \pm 0.02^{\mathrm{B}}$ & $0.12 \pm 0.00^{\mathrm{A}}$ & $0.21 \pm 0.00^{\mathrm{B}}$ & ND & $11.82 \pm 0.02^{\mathrm{abF}}$ & \\
\hline & 18 & $1.09 \pm 0.00^{\mathrm{B}}$ & $0.57 \pm 0.00^{\mathrm{A}}$ & $0.06 \pm 0.00^{C}$ & $0.06 \pm 0.00^{\mathrm{B}}$ & $0.11 \pm 0.00^{C}$ & ND & $31.36 \pm 0.22^{\mathrm{bB}}$ & \\
\hline & 21 & $1.21 \pm 0.03^{\mathrm{A}}$ & $0.40 \pm 0.01^{\mathrm{C}}$ & $0.50 \pm 0.01^{\mathrm{A}}$ & $0.03 \pm 0.02^{\mathrm{C}}$ & $0.27 \pm 0.02^{\mathrm{A}}$ & ND & $22.32 \pm 0.61^{\mathrm{c} C}$ & \\
\hline
\end{tabular}

a-cMeans with different superscripts within the same time group are significantly different $(p<0.05)$. A-FMeans with different superscripts within the same size group are significantly different $(p<0.05)$. ${ }^{1)}$ After cryogenic milling of ginseng samples with different conditions, the samples were classified by their particle sizes. One treatment condition in each size range was then selected, and samples selected by particle size were roasted and pulverized by cryogenic milling.

\section{Conclusions}

The purpose of this study was to decrease the particle size of ginseng by roasting and cryogenic milling and eventually increase the water solubility and physiological activity of ginseng.

Ginseng samples were roasted for different times of 9-21 min and ground to different sizes (1050 , and $>50 \mu \mathrm{m}$ ) by cryogenic milling. All roasted samples indicated significantly smaller particle sizes compared with the control (not roasted) based on D [3,2] $(p<0.05)$. The particle sizes of the roasted samples showed a decreasing pattern until $15 \mathrm{~min}$ of roasting. However, with roasting for over $18 \mathrm{~min}$, the particle size of the ginseng powder was increased due to flocculation. WSI values indicated an increasing pattern as the particle size decreased except for samples roasted at over 18 min. As for the results of antioxidant activity and TPC, samples with 10-20 $\mu \mathrm{m}$ size demonstrated higher values than those for over $50 \mu \mathrm{m}$-sized samples. The total polysaccharide contents of 10-20 $\mu \mathrm{m}$-sized samples revealed significantly higher values than for over $50 \mu \mathrm{m}$-sized samples with an exception of samples roasted for $21 \mathrm{~min}(p<0.05)$. However, acidic polysaccharides did not show any trend according to particle size. Ginsenoside contents showed no tendency according to particle size. Based on roasting time, the WSI showed higher values up to $15 \mathrm{~min}$ of roasting compared to the control (not roasted) $(p<0.05)$. Antioxidant activity and TPC also showed an increasing trend with increasing roasting time. Total polysaccharides showed the highest values when the samples were roasted for $15 \mathrm{~min}$ except for the particles with 10-20 $\mu \mathrm{m}$ size. Acidic polysaccharide values showed no tendency depending on roasting time, but showed significantly higher values than did the control 
(not roasted). Ginsenoside did not show any pattern depending on roasting time, but the ginsenoside contents of roasted samples with over $20 \mu \mathrm{m}$ size were higher than the control except in samples roasted for $15 \mathrm{~min}$. This study proves that roasting and cryogenic milling for certain times have a positive effect such as reduction of particle size, increased WSI, and increased physiological activity in the manufacture of ginseng powder.

Author Contributions: H. Jeong performed the experiments and wrote original draft; D. H. Park performed the experiments; H. G. Seo and M.-J. Choi provided technical and material support; Y. Cho interpreted the data and critically revised the manuscript.

Funding: This work was supported by Korea Institute of Planning and Evaluation for Technology in Food, Agriculture and Forestry (iPET) through High Value-added Food Technology Development Program, funded by Ministry of Agriculture, Food and Rural Affairs (MAFRA) (318028-03). This work was also supported by the KU Research Professor Program of Konkuk University.

Conflicts of Interest: The authors declare no conflict of interest.

\section{References}

1. Yun, T.K. Brief introduction of Panax ginseng C.A. Meyer. J Korean Med Sci 2001, 16, S3-S5. DOI: 10.3346/jkms.2001.16.S.S3.

2. Park, S.Y.; Lee, K.Y.; Cho, Y.J.; Park, B.K.; Kim, K.J.; Lee, N.R.; Kim, D.G.; Kim, Y.H.; Hahn, T.W. Efficacy of orally administered ginseng stem and leaf in chickens. Korean J. Vet. Res. 2015, 55(1), 1-7. DOI: 10.14405/kjvr.2015.55.1.1.

3. Li, X.G. Studies on the transforming mechanism of amino acid components in ginseng in the course of ginseng process. J. Ginseng Res. 1992, 16(1), 64-67.

4. Kang, K.S.; Yokozawa, T.; Yamabe, N.; Kim, H. Y.; Park, J.H. ESR study on the structure and hydroxyl radical-scavenging activity relationships of ginsenosides isolate from Panax ginseng C. A. Meyer. Biol. Pharm. Bull. 2007, 30(5), 917-921. DOI: 10.1248/bpb.30.917.

5. Park, C.K.; Jeon, B.S.; Yang, J.W. The chemical components of Korean ginseng. Food Ind Nutr. 2003, 8(2), 1023.

6. Lee, J.H.; Choi, K.H.; Sohn, E.H.; Jang, K.H. Quality characteristics and ginsenosides composition of ginseng-Yakju according to the particle size of ginseng powder. Nutr. Food Sci Prev. 2013, 18(4), 234-241. DOI: 10.3746/pnf.2013.18.4.234.

7. Choi, K.T. Botanical characteristics, pharmacological effects and medicinal compo-nents of Korean Panax ginseng C A Meyer. Acta Pharmacologica Sinica 2008, 29(9), 1109-1118. DOI: 10.1111/J.1745-7254.2008.00869.x.

8. Lu, J.M.; Yao, Q.; Chen, C. Ginseng compounds: an update on their molecular mechanisms and medical applications. Curr. Vasc. Pharmacol. 2009, 7(3), 293-302. DOI: 10.2174/157016109788340767.

9. Choi, K.O.; Lee, I.; Park, S.Y.R.; Kim, D.E.; Lim, J.D.; Kang, W.S.; Ko, S.H. Ultrafine Angelica gigas powder normalizes ovarian hormone levels and has antiosteoporosis properties in ovariectomized rats: particle size effect. J. Med. Food 2012, 15(10), 863-872. DOI: 10.1089/jmf.2011.2047.

10. Suh, C.S.; Chun, J.K. Relationships among the roasting conditions, colors and extractable solid content of roasted barley. Korean J. Food Sci. Technol. 1981, 13(4), 334-339.

11. Redgwell, R.J.; Trovato, V.; Curti, D. Cocoa bean carbohydrates: roasting induced changes and polymer interactions. Food Chem. 2003, 80, 511-516. DOI: 10.1016/S0308-8146(02)00320-5. 
12. Redgwell, R.J.; Trovato, V.; Curti, D.; Fischer, M. Effect of roasting on degradation and structural features of polysaccharides in Arabica coffee beans. Carbohydr. Res. 2002, 337(5), 421-431. DOI: 10.1016/S00086215(02)00010-1.

13. Saklara, S.; Unganb, S.; Katnasc, S. Microstructural changes in hazelnuts during roasting. Food Res Int 2003, 36(1), 19-23. DOI: 10.1016/S0963-9969(02)00103-5.

14. Jeon, E.J.; Kim, K.Y.; Lee, J.E.; Catherine, W.; Kwon, J.H. Monitoring of Roasting Conditions for the Functional Properties of Lateral Root of Red Ginseng. Korean J. Food Preserv. 2008, 15(3), 396-404.

15. Park, M.H.; Kim, K.C. Changes in Physicochemical Components of Ginseng Marc by Roasting Process. Korean J. Ginseng Sci. 1995, 19(2), 144-152.

16. Sivetz, M.; Desrosier, N.W. Coffee technology. United States Department of Agriculture National Agricultural Library. Westport, CT: Avi, USA, 1979; pp 716.

17. Yoon, S.K.; Kim, W.J. Effects of roasting conditions on quality and yields of barley tea. Korean J. Food Sci. Technol. 1989, 21(4), 575-582.

18. Lee, B.G.; Lee, K.Y.; Jorge, S.; Jorge, R.; Baek, H.; Min, J.H.; Kang, W.S. Ultrafine powderization using low temperature turbo mill to improve water solubility of red ginseng powder. In 2012 12th IEEE International Conference on Nanotechnology (IEEE-NANO), 2012, 1-4. DOI: 10.1109/NANO.2012.6321975.

19. Manohar, B.; Sridhar, B.S. Size and shape characterization of conventionally and cryogenically ground turmeric (Curcuma domestica) particles. Powder Technol 2001, 120(3), 292-297. DOI: 10.1016/S00325910(01)00284-4.

20. Meghwal, M.; Goswami, T.K. Evaluation of size reduction and power requirement in ambient and cryogenically ground fenugreek powder. Advanced Powder Technology 2013, 24(1), 427-435. DOI: 10.1016/j.apt.2012.09.005.

21. Lee, S.B.; Yoo, S.H.; Ganesan, P.; Kwak, H.S. Physicochemical and antioxidative properties of Korean nanopowdered white ginseng. Food Sci. Technol 2013, 48, 2159-2165. DOI: 10.1111/IJFS.12200.

22. Blois, M.S. Antioxidant determinations by the use of a stable free radical. Nature 1958, 181, 1199-1200. DOI: 10.1038/1811199a0.

23. Ough, C.S.; American, M.A. Methods for analysis of musts and wine; Wiley \& Sons: New York, NY, USA, 1988; pp 176-180.

24. Dubois, M.; Gillers, K.A.; Hamilton, J.K.; Rebers, P.A.; Smith, J. Colorimetric method for determination of sugar and related substance. Anal. Chem. 1956, 28(3), 350-356. DOI: 10.1021/ac60111A017.

25. Do, J.H.; Lee, H.O.; Lee, S.K.; Jang, J.K.; Lee, S.D.; Sung, H.S. Colorimetric Determination of Acidic Polysaccharide from Panax ginseng, its Extraction Condition and Stability). J. Ginseng Res. 1993, 17(2), 139144.

26. Kim W.; Choi S.; Kerr W.; Johnson J.; Gaines, C. Effect of heating temperature on particle size distribution in hard and soft wheat flour. J. Cereal Sci. 2004, 40(1), 9-16. DOI: 10.1016/j.jcs.2004.04.005.

27. Im, G.Y.; Jang, S.Y.; Jeong, Y.J. Quality characteristics of panax ginseng C. A. Meyer with steaming heat and wet grinding conditions. J Korean Soc Food Sci Nutr. 2010, 39(7), 1005-1010. DOI: 10.3746/jkfn.2010.39.7.1005.

28. Lee, J.S.; Lee, H.S. Effect of grinding methods on particle size and crystalline structure of copper phthalocyanine. J Ind Eng Chem. 2007, 18(1), 41-47. 
29. Jo, G.S., Sin, J.S.; Kim, J.H. Measurement of particle size and particle size distribution. Polymer Sci Technol 2004, 15(2), 198-208.

30. Uhm, Y.R.; Kim, J.W.; Jung, J.W.; Rhee, C.K. The fabrication of PVA polymer coated on the surface of B4C nanocomposite by high energy ball mill. J. Korean Powder Metall. 2009, 16(2), 110-114. DOI: 10.4150/KPMI.2009.16.2.110.

31. Kim, H.Y.; Seo, H.I.; Ko, J.Y.; Kim, J.I.; Lee, J.S.; Song, S.B.; Jung, T.W.; Kim, K.Y.; Kwak, D.Y.; Oh, I.S.; Jeong, H.S.; Woo, K.S. Physicochemical characteristics of the sorghum(Sorghum bicolor L Moench) powder following low temperature-microparticulation. Koran J. Food $\mathcal{E}$ Nutr. 2012, 25(3), 656-663. DOI: 10.9799/ksfan.2012.25.3.656.

32. Lee, S.B.; Yoo, S.H.; Ganesan, P.; Kwak, H.S. Physicochemical and antioxidative properties of Korean nanopowdered white ginseng. Food Sci. Technol 2013, 48, 2159-2165. DOI: 10.1111/ijfs.12200.

33. Kim, E.K.; Jeong, Y.H.; Gu, S.Y.; Song, K.Y.; Kim, I.Y.; Kim, K.Y. Physicochemical characteristics of Brazilian coffea arabica cv. Catuai coffee extracts with different roasting conditions. J Korean Soc Food Sci Nutr. 2019, 48(7), 748-756. DOI: 10.3746/jkfn.2019.48.7.748.

34. Hemansson, A. "Gel characteristics-structure as related to texture and water binding of blood plasma gels". J. Food Sci. 1982, 47(6), 1965-1972.

35. Guha, M.; Ali, S.Z.; Bhattacharya, S. Twin-screw extrusion of rice flour without a die: effect of barrel temperature and screw speed on extrusion and extrudate characteristics. J. Food Eng. 1997, 32(3), 251-267. DOI: 10.1016/S0260-8774(97)00028-9.

36. Zavareze, E.D.R.; Dias, A.R.G. Impact of heat-moisture treatment and annealing in starches: A review. Carbohydr. 2011, 83(2), 317-328. DOI: 10.1016/j.carbpol.2010.08.064.

37. Cho, Y.J. Antioxidant, angiotensinconverting enzyme and xanthin oxidase inhibitory activity of extracts from Saururus chinensis leaves by ultrafine grinding. Korean J. Food Preserv. 2014, 21(1), 75-81. DOI: 10.11002/kjfp.2014.21.1.75.

38. Kim, K.H.; Lee, I.H.; Lee, H.S.; Park, J.K. R\&D trend and information analysis of nanoparticles. Pros Ind Chem. 2013, 6, 46-61

39. Kim, C.S.; Kim, C.S.; Kim, H.I. Physicochemical properties of non-waxy rice flour affected by grinding methods and steeping times. J Korean Soc Food Sci Nutr. 2009, 38, 1076-1083. DOI: 10.3746/jkfn.2009.38.8.1076.

40. Song, Y.B.; Lee, K.S.; Lee, M,S.; Kim, A.J. Bioactivity changes in mung beans according to the roasting time. Korean J. Food \& Nutr. 2013, 26(3), 502-507. DOI: 10.9799/ksfan.2013.26.3.502.

41. Seong, B.J.; Kim, S.I.; Jee, M.G.; Kim, S.D.; Kwon, A.R.; Kim, H.H.; Hwang, Y.G.; Lee, K.S. Physicochemical characteristics according to the roasting conditions and grinding grade for the development of drip type red ginseng. J Korean Soc Food Sci Nutr 2018, 47(3), 309-319. DOI: 10.3746/jkfn.2018.47.3.309.

42. Cha, S.M.; Son, B.Y.; Lee, J.S.; Baek, S.B.; Kim, S.L.; Ku, J.H.; Hwang, J.J.; Song, B.H.; Woo, S.H.; Kwon, Y.U.; Kim, J.T. Effect of particle size on physico-chemical properties and antioxidant activity of corn silk powder. Korean J. Crop Sci. 2012, 57(1), 41-50. DOI: 10.7740/kjcs.2012.57.1.041.

43. Durmaz, G.; Alpaslan, M. Antioxidant properties of roasted apricot (Prunus armeniaca L.). Kernel. Food Chem. 2007, 100(3), 1177-1181.

44. Kim, J.S.; Kang, O.J.; Gweon, O.C. Comparison of phenolic acids and flavonoids in black garlic at different thermal processing steps. J. Funct. Foods 2013, 5(1), 80-86. DOI: 10.1016/j.jff.2012.08.006. 
45. Chung, H.S.; Chung, S.K.; Youn, K.S. Effects of roasting temperature and time on bulk density, soluble solids, browning index and phenolic compounds of corn kernels. J Food Process Pres. 2011, 35(6), 832-839. DOI: 10.1111/j.1745-4549.2011.00536.x.

46. Cho, K.L; Woo, H.J.; Lee, I.S.; Lee, J.W.; Cho, Y.C.; Lee, I.N.; Chae, H.J. Optimization of Enzymatic Pretreatment for the Production of Fermented Ginseng using Leaves, Stems and Roots of Ginseng. J. Ginseng Res. 2010, 34(1), 68-75. DOI: 10.5142/JGR.2010.34.1.068.

47. Cho, C.W.; Kim, S.W.; Rho, J.H.; Rhee, Y.K. Extraction characteristics of saponin and acidic polysaccharide based on the red ginseng particle size. J. Ginseng Res. 2008, 32(3), 179-186. DOI: 10.5142/JGR.2008.32.3.179.

48. Robert, J.; Redgwell.; Veronique, T.; Delphine, C.; Monica, F. Effect of roasting on degradation and structural features of polysaccharides in Arabica coffee beans. Carbohydr. Res. 2002, 337(5), 421-431. DOI: 10.1016/S0008-6215(02)00010-1.

49. Lee, J.W.; Do, J.H. Extraction condition of acidic polysaccharide from Korean red ginseng marc. J. Ginseng Res. 2002, 26(4), 202-205. DOI: 10.5142/JGR.2002.26.4.202.

50. Zhang, X.; Yu, L.; Bi, H.; Li, X.; Ni, W.; Han, H.; Li, N.; Wang, B.; Zhou, Y.; Tai, G. Total fractionation and characterization of the water soluble polysaccharides iso-lated from panax ginsen C.A. Meyer. Carbohydr. Polym. 2009, 77(3). 544-552. DOI: 10.1016/j.carbpol.2009.01.034. 